\title{
1 Antimicrobial exposure in sexual networks drives divergent 2 evolution in modern gonococci
}

4 Leonor Sánchez-Busó ${ }^{1}$, Daniel Golparian², Jukka Corander ${ }^{1,3,4}$, Yonatan H. Grad ${ }^{5,6}$,

5 Makoto Ohnishi ${ }^{7,8}$, Rebecca Flemming ${ }^{9}$, Julian Parkhill ${ }^{1}$, Stephen D. Bentley ${ }^{1}$,

6 Magnus Unemo ${ }^{2}$, Simon R. Harris ${ }^{1 *}$

$8{ }^{1}$ Infection Genomics, Wellcome Sanger Institute, Wellcome Genome Campus,

9 Hinxton, Cambridgeshire, UK.

$10{ }^{2}$ WHO Collaborating Centre for Gonorrhoea and other Sexually Transmitted

11 Infections, National Reference Laboratory for Sexually Transmitted Infections,

12 Department of Laboratory Medicine, Clinical Microbiology, Faculty of Medicine and

13 Health, Örebro University, Örebro, Sweden.

$14{ }^{3}$ Helsinki Institute for Information Technology, Department of Mathematics and

15 Statistics, University of Helsinki, 00014, Helsinki, Finland.

$16{ }^{4}$ Department of Biostatistics, University of Oslo, 0317, Oslo, Norway.

17 Department of Immunology and Infectious Diseases, Harvard TH Chan School of 18 Public Health, Boston MA, USA.

$19{ }^{6}$ Division of Infectious Diseases, Brigham and Women's Hospital, Harvard Medical 20 School, Boston MA, USA.

$21{ }^{7}$ Department of Bacteriology I, National Institute of Infectious Diseases, Tokyo, 22 Japan.

$23{ }^{8}$ Antimicrobial Resistance Research Center, National Institute of Infectious Diseases, 24 Tokyo, Japan.

$25{ }^{9}$ Faculty of Classics, University of Cambridge, Cambridge, UK. 


\section{Abstract:}

31 The sexually transmitted pathogen Neisseria gonorrhoeae is regarded as being on the

32 way to becoming an untreatable superbug. Despite its clinical importance, little is

33 known about its emergence and evolution, and how this corresponds with the

34 introduction of antimicrobials. We present a genome-based phylogeographic analysis

35 of 419 gonococcal isolates from across the globe. Results indicate that modern

36 gonococci originated in Europe or Africa as late as the $16^{\text {th }}$ century and subsequently

37 disseminated globally. We provide evidence that the modern gonococcal population

38 has been shaped by antimicrobial treatment of sexually transmitted and other infections,

39 leading to the emergence of two major lineages with different evolutionary strategies.

40 The well-described multi-resistant lineage is associated with high rates of homologous

41 recombination and infection in high-risk sexual networks where antimicrobial

42 treatment is frequent. A second, multi-susceptible lineage associated with heterosexual

43 networks, where asymptomatic infection is more common, was also identified, with

44 potential implications for infection control. 
53 Almost 360 million curable sexually transmitted infections (STIs) are estimated to

54 occur globally each year, with Neisseria gonorrhoeae, the causative agent of 55 gonorrhoea, infecting approximately 78 million $^{1}$. The highest gonorrhoea burden is 56 among men, although problematic infections are more common in women for whom 57 the early stages of infection are often asymptomatic. Unresolved urogenital infections can lead to severe complications and sequelae, such as reproductive problems including infertility, serious eye infections in newborns, and enhanced transmission of HIV ${ }^{2}$. The emergence and proliferation of gonococci with reduced susceptibility to front-line antimicrobials such as extended-spectrum cephalosporins (ESCs; cefixime and ceftriaxone) and azithromycin have contributed to, although do not completely explain,

63 the increase in incidence of gonorrhoea. Resistance to dual therapy (injectable ceftriaxone plus oral azithromycin), the current recommended treatment in many countries, is fortunately rare ${ }^{3}$, however, ceftriaxone resistance has been reported from all continents and azithromycin resistance is on the increase globally ${ }^{4}$, raising fears that the effectiveness of this regimen will be short-lived. Much of the focus of gonococcal control is on particular high-risk sexual networks that often partake in unprotected sex with multiple partners, particularly sex workers and men who have sex with men (MSM) but also young heterosexuals. These groups are more frequently exposed to

71 both infection and antimicrobial treatment, which has led to these networks being the suspected drivers of antimicrobial resistance (AMR $)^{5}$. However, AMR is not the only

73 factor driving the recent success of $N$. gonorrhoeae. Dual therapy is effective against 74 the vast majority of infections, yet since its introduction, gonorrhoea infections have 75 continued to increase in most settings ${ }^{6}$.

76 Georges Luys famously opened his medical textbook on gonorrhoea with the statement 77 that 'Gonorrhoea is as old as mankind' ${ }^{7}$. However, despite $N$. gonorrhoeae often being 
described as an ancient pathogen, there are no clear descriptions of a disease like modern gonorrhoea in the ancient sources. Some compatible symptoms do appear in the medical literature of classical Greece and Rome, but nothing decisive, and the presence or absence of modern gonorrhoea in the ancient Mediterranean has been much debated as a result ${ }^{8}$. Early modern terms like 'the clap', 'the pox', or 'the venereal disease' also covered a range of conditions, and it was not until 1879 that Alfred Neisser identified the bacteria that now bears his name ${ }^{9}$. AMR in gonorrhoea became apparent soon after antimicrobials were first introduced for its treatment. Sulfonamide therapy was initially successful, but shortly after its introduction in 1935 the emergence of resistance curtailed its effectiveness. Subsequently, gonococci have developed resistance to all available treatments, including penicillins, tetracyclines, quinolones, macrolides and $\mathrm{ESCs}^{2}$. One characteristic of $N$. gonorrhoeae that has played an important role in its rapid gain and spread of AMR is its ability to exchange DNA via homologous recombination both within its own species and with another Neisseria. For example, mosaic penicillin-binding protein 2 (PBP2; encoded by the penA gene) alleles gained via recombination have been key in the emergence of resistance to $\mathrm{ESCs}^{10,11}$ which led to the replacement of cefixime as the first-line treatment for gonorrhoea. The first ceftriaxone resistant mosaic penA genotype was seen in an isolate from a pharyngeal infection in a female sex worker in Japan in $2009^{12}$, but similar mosaics

97 were subsequently soon seen worldwide ${ }^{2,13}$. In fact, a number of resistances have first

98 been identified in Japan, leading to the hypothesis that most AMR gonorrhoea 99 originates there, or elsewhere in the WHO Western Pacific Region².

100 Whole-genome sequencing has been successfully used to reveal the origins, global 101 spread and population structure of several human pathogens ${ }^{14}$. However, gonococcal 102 genome sequencing has mostly targeted specific populations and outbreaks ${ }^{15-19}$. Here, 
103 we report the findings of a global genomic study of $419 \mathrm{~N}$. gonorrhoeae isolates

104 spanning five continents and more than 50 years, including varying susceptibilities to

105 important antimicrobials. Our aim was to elucidate when and where modern gonococcal

106 populations emerged, evolved and dispersed, and how antimicrobial usage and

107 transmission in different sexual networks has influenced their population dynamics.

\section{Modern gonococcus not 'as old as mankind'}

109 Our collection spans a period of more than 50 years $(1960-2013)$ and 58 countries

110 from five continents (Supplementary Table 1, Figure 1). A population-level analysis

111 revealed a high level of admixture among $N$. gonorrhoeae with no significant

112 differentiation between continents (Supplementary Table 2), with the exception of

113 Africa (Extended Data Figure 1, Supplementary Tables 3-5). We estimated the

114 substitution rate for the non-recombining section of the genomes in the collection

115 (Extended Data Figure 2, panel a) to be $3.74 \mathrm{E}^{-06}$ substitutions/site/year CI (confidence

116 interval) $\left[3.39 \mathrm{E}^{-06}-4.07 \mathrm{E}^{-06}\right]$, which is similar to previous reports ${ }^{15,17}$ and comparable

117 to rates calculated for other bacteria ${ }^{20}$. The time of the most recent common ancestor

118 (tMRCA) was estimated to be around the $16^{\text {th }}$ century $(1589$, CI [1544 - 1623]) (Figure

119 2). Although high rates of recombination can lead to underestimation of tMRCAs to

120 some extent, these results are strongly at odds with the hypothesis that modern

121 gonorrhoea has existed as long as mankind and cast further doubt on the ascribing of

122 historical descriptions of gonorrhoea-like symptoms to infection with 'modern'

123 gonococci.

124 Despite modern gonococci being globally mixed, we found strong evidence of historic 125 geographical separation, suggesting rapid mixing of populations is a relatively recent 126 phenomenon. A phylogeographic analysis ascribed the origin of our collection to 127 Europe (60.9\% proportion of inferred ancestry). However, when corrected for biases in 
128 the number of samples from each continent, in part by complementing using isolates

129 from a US study ${ }^{15}$, there was support for an African (90.7\% proportion of inferred

130 ancestry) origin (Extended Data Figure 3, panel a and b and Supplementary Table 6).

131 From this African root, we identified a number of change-points in the continental

132 distribution of isolates across the tree (Extended Data Figure 3, panels c-d). The most

133 significant change-point separated a basal lineage containing a high proportion $(68.2 \%$,

134 30/44) of African isolates and a lineage containing a high proportion of Asian isolates

$135(92.6 \%, 137 / 148)$, despite the temporal sampling from the two continents being similar

136 (Extended Data Figure 3, panels e-f). When combined with the dating, this can be

137 interpreted as an early introduction of the modern gonococcus population into Asia

138 (1617, CI [1578 - 1649], Figure 2) soon after its emergence in Africa or Europe. More

139 recently, many re-introductions into the rest of the world have occurred from this Asian

140 lineage, contributing to the highly mixed population observed today.

\section{Emergence of antimicrobial resistant gonorrhoea}

142 Interestingly, MICs for six antimicrobials (Extended Data Figure 4) and the occurrence

143 of genetic AMR determinants were significantly higher among the isolates belonging

144 to the lineage that arose after the phylogeographic breakpoint representing the initial

145 introduction into Asia (Student's t p-value $<0.0001$ ) (Figures 3 and 4c). We will

146 therefore refer to the 298 after the breakpoint as lineage A and the 121 isolates before

147 the breakpoint as lineage B.

148 Two AMR determinants, folP R228S, which reduces susceptibility to sulfonamides,

149 and rpsJ V57M, which reduces susceptibility to tetracyclines, were carried by a large

150 proportion of isolates, especially in lineage A (Supplementary Table 1, Figure 3). Fifty-

151 one isolates contained a mosaic penA allele ${ }^{21}$, which are generally believed to be the

152 result of recombination events with other Neisseria species. We identified three 
153 independent gains of mosaic alleles, all in lineage A. In a clade of 59 isolates with

154 MLST ST1901, a first recombination event replaced the wild type allele with a penA10

155 allele and a subsequent event replaced penA10 with penA34. These two alleles differ

156 by 16 SNPs and a codon insertion in the last 105 bases of the nucleotide sequence. Two

157 isolates in this clade exhibited high Minimum Inhibitory Concentrations (MICs) for 158 both cefixime (3-4 $\mu \mathrm{g} / \mathrm{ml})$ and ceftriaxone $(2 \mu \mathrm{g} / \mathrm{ml})$ (Extended Data Figure 5), and 159 these were found to possess a penA42, which is a single SNP (A501P) variant from 160 penA34 ${ }^{22,23}$. In another lineage, associated with MLST ST7363, most isolates possessed 161 the penA10 alleles, but we again observed a case of replacement with penA34. Only 162 one isolate carried the A2045G 23S rRNA mutation (A2059G in Escherichia coli 163 nomenclature) that confers high-level resistance to azithromycin. Six carried the low164 level resistance C2597T 23S mutation (C2611T in E. coli nomenclature). As expected, 165 isolates carrying a tetM gene on a conjugative plasmid and/or the blaTEM plasmid 166 showed increased resistance to tetracyclines and penicillin, respectively (Extended 167 Data Figure 5). Strikingly, these two plasmids co-localized far more frequently than 168 expected (Fisher's test p-value $<0.0001$ ), possibly reflecting the mobilization of 169 pBlaTEM by the pConjugative plasmid $^{24}$, and were completely absent from ESC170 resistant isolates (Figure 3). The plasmid-encoded resistances showed no significant 171 difference in prevalence in lineages $\mathrm{A}$ or $\mathrm{B}$ (tetM $\mathrm{p}$-value $=0.92$, bla $\mathrm{TEM} \mathrm{p}$-value $=$ 172 0.35). In contrast, of the 29 chromosomally-mediated resistance substitutions 173 examined, 18 were significantly associated with clade A (Figure 3). Importantly, based 174 on our phylogenetic dating, the majority of occurrences of these 29 determinants were 175 estimated to have been acquired after the introduction of the antimicrobial against 176 which they act (Extended Data Figure 6). The Gonococcal Genomic Island (GGI) was 
177 found in $277(67 \%)$ isolates (Extended Data Figure 7), but showed no clear association 178 with AMR.

\section{Two strategies for gonococcal success}

180 Overall, our data show far fewer gains of chromosomally-encoded AMR determinants

181 in lineage B compared to A (Extended Data Figure 8, panel a). Since these determinants

182 primarily spread through the population via homologous recombination, such 183 differences could be explained by differences in recombination frequency. To assess 184 this, we compared the proportion of homoplasic sites, an indicator of recombination, in 185 the terminal branches of the phylogenetic tree in the two lineages. This confirmed a 186 significantly higher proportion in clade A, particularly for short branches, which 187 represent very recent evolution (t-test $\mathrm{p}$-value $<0.005$; Figures $4 \mathrm{a}-\mathrm{b}$ and Extended Data 188 Figure 9). Similarly, the proportion of clustered SNPs, another signal of recombination, 189 was also higher on the terminal branches in lineage $\mathrm{A}(\mathrm{p}$-value $<0.05)$.

190 One explanation for such differences could be opportunity. For recombination to occur, 191 donor and recipient bacteria must co-localise. Thus, recombination between gonococci 192 would be expected to occur more frequently in high-risk host populations where 193 coinfection with other STIs and pharyngeal infections, which allow access to 194 commensal Neisseria species, are more common. For gonorrhoea, these risk-groups 195 include MSM, sex workers and some groups of young heterosexuals. These groups are 196 also more likely to be exposed to repeated antimicrobial therapy for gonorrhoea and 197 other STIs ${ }^{5}$. Unfortunately, due to limitations in availability of data on patient sexual 198 behaviour, we could not adequately assess association of the lineages to risk factors in 199 our dataset. However, we could analyse the distribution of the gender of the patients 200 from which the isolates were taken. To increase the power of the analysis, we included 201376 isolates from two North-American genomic studies ${ }^{16,25}$, to give a set of 639 isolates 
with complete gender information. Strikingly, lineage B included a significantly higher

204 (Figure 4d and Extended Data Figure 8, panel b), which would suggest that B is more

205 closely associated with heterosexuals. Corroborating this, data from a 2013 European-

206 wide structured survey of over $1,000 \mathrm{~N}$. gonorrhoeae isolates ${ }^{26}$ showed a similar

207 pattern. Lineage B isolates were strongly associated with reduced MICs and female

208 patients $(61 / 214,28.5 \%$ of lineage B isolates were from women vs $100 / 821,12 \%$ of

209 lineage A; p-value < 0.0001), and more importantly, of the patients that reported sexual

210 orientation, $78.3 \%(94 / 120)$ of isolates in lineage B were from heterosexuals, in contrast

211 to $52.6 \%(200 / 380)$ in A (p-value < 0.0001) (Extended Data Figure 10). Particular

212 sublineages within lineage B appear particularly strongly associated with

213 heterosexuals ${ }^{26}$. We suspect lineage $\mathrm{A}$, being associated with higher-risk populations,

214 does have greater opportunity for recombination, which may explain the observed

215 higher recombination rate. However, transmission between low and high-risk

216 populations is common within lineage A, so we suspect opportunity is not the only

217 explanation for the differential recombination rate in the two lineages. The observation

218 that plasmid-born resistances do not show the same difference in frequency between

219 the two lineages also supports this view.

\section{Discussion}

221 Gonorrhoea is one of the most clinically important STIs worldwide. Its rapid mode of

222 transmission, especially among high-risk groups, and the emergence of resistance to

223 many antimicrobials, has made the control of $N$. gonorrhoeae of primary importance

224 for public health. In recent years there has been an understandable focus on AMR

225 gonorrhoea, with resistance to all classes of antimicrobials used to treat the infection 
226 having been reported ${ }^{2}$. However, the increase in prevalence of gonorrhoea has

227 continued in many settings ${ }^{6}$ despite resistance to dual therapy being extremely rare.

228 Our genomic analysis revealed a contemporary global population with little

229 geographical structure, suggesting rapid recent intercontinental transmission is

230 occurring. In particular, introductions from Asia into the rest of the world appear

231 common, consistent with the observation that a number of recent resistant gonococcal

232 clones have emerged from this region ${ }^{2}$. The one exception was Africa, where the

233 sampled gonococcus was less diverse, potentially reflecting the relatively low rates of

234 human migration with other continents ${ }^{27}$. However, our African sample size was small

235 due to limited availability of isolates, so further study is required in this area.

236 We estimated an origin of modern gonococci in the $16^{\text {th }}$ century $(1544-1623)$, which 237 contrasts with historical interpretations of gonorrhoea as an ancient disease. Although

238 we are keen to stress that high rates of recombination make accurate estimates difficult,

239 and our confidence intervals are probably too narrow, this dating suggests that ancient

240 accounts of gonorrhoeal-like symptoms may have been caused by other pathogens, or

241 are evidence of an ancient $N$. gonorrhoeae population distinct from that observed today.

242 It certainly disputes the view that the disease we now know as gonorrhoea is 'as old as

243 mankind'. The $16^{\text {th }}$ century was, nonetheless, an opportune time for the global

244 dissemination of pathogens. It was a period of early modern globalization marked by

245 the initiation and intensification of many intercontinental trade links, particularly by

$246 \mathrm{sea}^{28}$. This period was of utmost importance for globalization due to an expeditious

247 increase in exchange of goods, including the import of new crops from the Americas to

248 Europe. Increased movement of people around the world also spawned local epidemics

249 and pandemics ${ }^{29}$, and may well have played an important role in the evolution of

250 modern gonorrhoea. A phylogeographic analysis using several subsampled set of 
251 strains from different continents to avoid bias placed the origin of the current global gonococcal population in Europe or Africa, and identified a subsequent introduction into Asia in the early $17^{\text {th }}$ century $(1578-1649)$, which expanded rapidly throughout

254 the continent. More recently this lineage has been repeatedly transmitted back to the 255 rest of the world.

256 A major finding is a strong association between isolates from the lineage that evolved 257 from this early introduction to Asia and the development of AMR. Nearly all isolates 258 in this lineage $\mathrm{A}$, and over $50 \%$ of those lineage $\mathrm{B}$, harboured resistance to 259 sulfonamides (folP R228S mutation) and tetracyclines (rpsJ V57M mutation). 260 Sulfonamides were the first antimicrobials introduced to treat gonorrhoea in 1935, with 261 initial efficacies of around 90\%. By the mid to late 1940s sulfonamide resistance was 262 common, and it was discarded as a treatment for gonorrhoea ${ }^{2}$. However, sulfonamides 263 are still widely used in combination with trimethoprim (TMP-SMZ) for prophylaxis in 264 HIV positive patients and to treat a variety of bacterial infections ${ }^{30}$. Doxycycline (a 265 tetracycline) is still sometimes used to treat gonococcal or presumptively non266 gonococcal urethritis/cervicitis and is the recommended treatment for 267 lymphogranuloma venereum ${ }^{31}$. We therefore suspect the high incidence of sulfonamide 268 and tetracycline resistance in modern gonorrhoea is due to historic treatment of the 269 disease itself followed by sustained use of these drugs for co-infections. The high 270 proportion of diverse circulating strains carrying the folP and $r s p J$ mutations could be 271 used as evidence that they were in the gonococcal population long before the 272 introduction of antimicrobials. However, this seems unlikely. More plausibly, the use 273 of sulfonamides and tetracyclines has produced a strong selective pressure over an 274 extended period of time, which has led to many independent acquisitions of resistance 275 mutations, or more likely, convergent gains of resistance via homologous 
recombination. In the more recombinogenic lineage $\mathrm{A}$, this has resulted in these mutations sweeping through the entire clade. Furthermore, other AMR determinants that have entered the gonococcal population more recently appear to be undergoing the same process, particularly in lineage A. The DNA gyrase A S91F substitution, which 280 provides resistance to ciprofloxacin, is one of many resistance mutations that show 281 extremely high levels of homoplasy in lineage A, consistent with rapid dissemination 282 via recombination. The mosaic penA alleles, which reduce susceptibility to ESCs are 283 another example. These elements were first described in N. gonorrhoeae around the 284 turn of the century, but have already been independently acquired by a number of A 285 sublineages, clearly showing that these mutations are transferring en masse via 286 recombination rather than by repeated de novo mutation. Lineage $\mathrm{B}$, on the other hand, 287 has remained susceptible to most antimicrobials. More generally, levels of homoplasy 288 and SNP clustering were found to be significantly higher in clade A, supporting the 289 hypothesis that higher rates of recombination in this lineage have facilitated its high 290 levels of AMR.

291 The rise of AMR gonorrhoea is generally assumed to have been facilitated by particular 292 demographics who partake in high-risk sexual behaviours, particularly unprotected sex 293 with multiple partners. These groups are also more often treated with antimicrobials 294 than the general population due to frequent infection. Concordantly, we found that 295 lineage A is associated with infection in MSM, one of the predominant risk groups, 296 while isolates from B are more rarely found in this demographic group. Thus, lineage 297 A isolates have the means (increased homologous recombination), motive (higher 298 antimicrobial exposure) and opportunity (higher rates of coinfection with commensal 299 Neisseria and other STIs) for recombination-driven gain of AMR. 
Most recent media attention and gonococcal genomics research has focussed on the

301 increasing levels of AMR in gonorrhoea. However, we have shown that a mostly

302 susceptible lineage of gonorrhoea is successfully persisting in lower-risk groups where

303 it is less likely to be exposed to antimicrobials. Notably, this lineage was associated

304 with heterosexual groups, and therefore with infections in women, where rates of asymptomatic infection are high. Turner et $a l .{ }^{32}$ showed, using a modelling approach, that in a situation where both resistant and susceptible strains are present in a population, high rates of asymptomatic infection, and therefore under-treatment, can allow susceptible isolates to survive and thrive. In such circumstances, rates of

309 susceptible infection can be hugely underestimated, potentially meaning that our 310 understanding of gonococcal prevalence, and rates of AMR may be biased.

311 Interestingly, the majority of our African samples were from lineage B, consistent with 312 epidemiological studies that describe a hidden epidemic of gonococcus in rural South 313 African women, in which $48 \%$ of cases were asymptomatic and another $50 \%$ were 314 symptomatic but not seeking care ${ }^{33}$. Similarly in Namibia, prevalence of asymptomatic 315 gonococcal infections in both men and women in rural villages are high ${ }^{34}$. This may 316 suggest that lineage B is associated with asymptomatic infection more fundamentally 317 than simply being more often found in women, reminiscent of Chlamydia trachomatis, 318 another highly prevalent STI which exhibits no AMR. In such a situation, if 319 compensatory mutations are not developed, gain of AMR determinants may be 320 detrimental as these elements may come with an associated general cost to fitness. Grad 321 et $a l^{35}$ reported, for example, that $23 \mathrm{~S}$ mutations associated with azithromycin 322 resistance led to reduced ESC MICs in isolates with mosaic penA alleles. Similarly, we 323 have observed that the tetM-containing pConjugative and pBlaTEM resistance 
324 plasmids are negatively associated with isolates with mosaic penA alleles, again

325 suggesting these elements are detrimental in combination.

326 In conclusion, in the first phylogeographic analysis of a global collection of gonococci

327 we have shown that although the modern gonococcal population is highly mixed, this

328 mixing is relatively recent. The modern population originated as late as the $16^{\text {th }}$ century, most likely in Europe or Africa, and an early single introduction into Asia led to a rapid spread throughout the continent and the rest of the world. Despite most recent focus

331 being on gonococcal AMR, we have demonstrated that $N$. gonorrhoeae has adapted to sexual networks with different risk profiles and exposures to antimicrobial treatment.

333 Modern global gonorrhoea can be divided into two lineages, which we term A (after

334 the phylogenetic breakpoint) and B (before the phylogenetic breakpoint). Lineage A 335 has gained and proliferated AMR determinants, aided by an increased rate of 336 recombination. These isolates are often transmitted in higher-risk networks, e.g. MSM, 337 where pharyngeal infections are more common and individuals are often exposed to 338 treatment for gonorrhoea and other STIs. Lineage B, however, has not gained AMR so 339 rapidly, with $26 \%$ of isolates containing no known AMR determinants, and is 340 potentially being silently transmitted in undertreated groups where levels of 341 asymptomatic infection are higher. Thus, our results have shown that the effect of 342 antimicrobial treatment on the gonococcal population has been more complex than 343 simply initiating an inexorable progression towards AMR.

\section{$344 \quad$ References}

3451 Newman, L. et al. Global Estimates of the Prevalence and Incidence of Four Curable Sexually Transmitted Infections in 2012 Based on Systematic Review and Global Reporting. PLoS One 10, e0143304, 
3492 Unemo, M., Del Rio, C. \& Shafer, W. M. Antimicrobial Resistance Expressed

350 by Neisseria gonorrhoeae: A Major Global Public Health Problem in the 21st

351 Century. Microbiol Spectr 4, doi:10.1128/microbiolspec.EI10-0009-2015

$352 \quad$ (2016).

3533 Fifer, H. et al. Failure of Dual Antimicrobial Therapy in Treatment of

$354 \quad$ Gonorrhea. N Engl J Med 374, 2504-2506, doi:10.1056/NEJMc1512757

$355 \quad$ (2016).

3564 Wi, T. et al. Antimicrobial resistance in Neisseria gonorrhoeae: Global

357 surveillance and a call for international collaborative action. PLoS Med 14,

$358 \quad$ e1002344, doi:10.1371/journal.pmed.1002344 (2017).

3595 Fingerhuth, S. M., Bonhoeffer, S., Low, N. \& Althaus, C. L. Antibiotic-

360 Resistant Neisseria gonorrhoeae Spread Faster with More Treatment, Not

361 More Sexual Partners. PLoS Pathog 12, e1005611,

362 doi:10.1371/journal.ppat.1005611 (2016).

3636 ECDC, European Centre for Disease Prevention and C. Annual

364 Epidemiological Report., (Stockholm: ECDC. Available from

365 http://ecdc.europa.eu/en/healthtopics/gonorrhoea/Pages/Annual-

366 Epidemiological-Report-2016.aspx, 2016).

3677 Luys, G. A textbook on gonorrhoea and its complications, translated by

368 Arthur Foerster (London: Baillière, Tindall and Cox, 1913). (1912).

3698 Grmek, M. in Diseases in the Ancient Greek World 142-149 (1989).

3709 Oriel, J. D. The Scars of Venus: A History of Venereology. (1994).

$371 \quad 10$ Spratt, B. G. Hybrid penicillin-binding proteins in penicillin-resistant strains

372 of Neisseria gonorrhoeae. Nature 332, 173-176, doi:10.1038/332173a0

$373 \quad$ (1988). 
37411 Ohnishi, M. et al. Is Neisseria gonorrhoeae initiating a future era of

375 untreatable gonorrhea?: detailed characterization of the first strain with high-

376 level resistance to ceftriaxone. Antimicrob Agents Chemother 55, 3538-3545, 377 doi:10.1128/AAC.00325-11 (2011).

37812 Ohnishi, M. Ceftriaxone-resistant Neisseria gonorrhoeae, Japan. Emerg Infect

379 Dis 17, 148-149, doi:10.3201/eid1701.100397 (2011).

38013 Lefebvre, B. et al. Ceftriaxone-Resistant Neisseria gonorrhoeae, Canada,

381 2017. Emerg Infect Dis 24, doi:10.3201/eid2402.171756 (2018).

38214 Popovich, K. J. \& Snitkin, E. S. Whole Genome Sequencing-Implications for 383 Infection Prevention and Outbreak Investigations. Curr Infect Dis Rep 19, 15, 384 doi:10.1007/s11908-017-0570-0 (2017).

38515 Grad, Y. H. et al. Genomic epidemiology of Neisseria gonorrhoeae with 386 reduced susceptibility to cefixime in the USA: a retrospective observational study. The Lancet Infectious Diseases 14, 220-226, doi:10.1016/s14733099(13)70693-5 (2014).

38916 Demczuk, W. et al. Whole-genome phylogenomic heterogeneity of Neisseria 390 gonorrhoeae isolates with decreased cephalosporin susceptibility collected in 391 Canada between 1989 and 2013. J Clin Microbiol 53, 191-200, 392 doi:10.1128/JCM.02589-14 (2015).

39317 De Silva, D. et al. Whole-genome sequencing to determine transmission of $394 \quad$ Neisseria gonorrhoeae: an observational study. Lancet Infect Dis, 395 doi:10.1016/S1473-3099(16)30157-8 (2016).

39618 Didelot, X. et al. Genomic Analysis and Comparison of Two Gonorrhea 397 Outbreaks. MBio 7, doi:10.1128/mBio.00525-16 (2016). 
39819 Chisholm, S. A. et al. An outbreak of high-level azithromycin resistant Neisseria gonorrhoeae in England. Sex Transm Infect, doi:10.1136/sextrans2015-052312 (2015).

40120 Duchene, S. et al. Genome-scale rates of evolutionary change in bacteria.

402 Microb Genom 2, e000094, doi:10.1099/mgen.0.000094 (2016).

40321 Demczuk, W. et al. Neisseria gonorrhoeae Sequence Typing for Antimicrobial Resistance, a Novel Antimicrobial Resistance Multilocus Typing Scheme for Tracking Global Dissemination of N. gonorrhoeae Strains. J Clin Microbiol 55, 1454-1468, doi:10.1128/JCM.00100-17 (2017).

40722 Cámara, J. et al. Molecular characterization of two high-level ceftriaxoneresistant Neisseria gonorrhoeae isolates detected in Catalonia, Spain. Journal of Antimicrobial Chemotherapy 67, 1858-1860, doi:10.1093/jac/dks 162 (2012).

41123 Unemo, M. et al. High-level cefixime- and ceftriaxone-resistant Neisseria gonorrhoeae in France: novel penA mosaic allele in a successful international clone causes treatment failure. Antimicrob Agents Chemother 56, 1273-1280, doi:10.1128/AAC.05760-11 (2012). mobilization of non-conjugative resistance plasmids by a 24.5 megadalton conjugative plasmid of Neisseria gonorrhoeae. J Gen Microbiol 125, 123-129,

41925 Demczuk, W. et al. Genomic Epidemiology and Molecular Resistance 
Harris, S. R. et al. Public health surveillance of multidrug-resistant clones of doi:https://doi.org/10.1016/S1473-3099(18)30225-1 (2018). Comparative Migration Studies 4, doi:10.1186/s40878-015-0015-6 (2016). Hopkins, A. G. Globalization in World History. (2002). (2001).

$433 \quad 31$ Wokowski, K. A. \& Bolan, G. A. Sexually Transmitted Diseases Treatment Guidelines, 2015. (Centers for Disease Control and Prevention, 2015). sexually transmitted infection on the evolution of the organism. Sex Transm

43833 Wilkinson, D. et al. Unrecognized sexually transmitted infections in rural South African women: a hidden epidemic. Bulletin of the World Health Organization 77, 22-28 (1999).

44134 Hazel, A., Ponnaluri-Wears, S., Davis, G. S., Low, B. S. \& Foxman, B. High prevalence of Neisseria gonorrhoeae in a remote, undertreated population of $443 \quad$ Namibian pastoralists. Epidemiology and Infection 142, 2422-2432, doi:10.1017/S0950268813003488 (2014).

44535 Grad, Y. H. et al. Genomic epidemiology of gonococcal resistance to extended 446 spectrum cephalosporins, macrolides, and fluoroquinolones in the US, 20002013. J Infect Dis, doi:10.1093/infdis/jiw420 (2016). 
448 Supplementary Information is linked to the online version of the paper at

449 www.nature.com/nature.

450 Acknowledgements: We thank Hien To and Olivier Gascuel for their help with the

451 LSD software, and the Pathogen Informatics group at the Wellcome Sanger Institute

452 for informatics support. We also thank Prof. Simon Szreter, Prof. Tim Bayliss-Smith

453 and Dr. Piers Mitchell from the University of Cambridge for interesting discussions

454 on the historical evidence of gonorrhoea infection. Japanese isolates were kindly

455 provided by Yuko Watanabe and Toshio Kuroki, Department of Microbiology,

456 Kanagawa Prefectural Institute of Public Health, Kanagawa, Japan. This work was

457 funded by Wellcome grant number 098051 and the Foundation for Medical Research

458 at Örebro University Hospital, Örebro, Sweden. JC was funded by the ERC grant no.

459745258 . YHG is supported by The Smith Family Foundation and NIH/NIAID grant

460 1R01AI132606-01.

\section{Author contributions:}

462 SRH, MU, SDB and JP conceived and managed the study. LSB and SRH analysed the

463 data and drafted the manuscript. DG, MU and MO cultured isolates and extracted

464 DNA. LSB, SRH, MU and YG interpreted the data. JC provided statistical analysis.

465 RF advised on historical interpretation. All authors contributed to the writing of the

466 manuscript.

467 Author information: Reprints and permissions information is available at

468 www.nature.com/reprints. The authors declare no competing interests. Readers are

469 welcome to comment on the online version of the paper. Publisher's note: Springer

470 Nature remains neutral with regard to jurisdictional claims in published maps and

471 institutional affiliations. Correspondence and requests for materials should be

472 addressed to S.R.H (sh16@ sanger.ac.uk). 
Figure and table legends

\section{Figure 1 | Geographic and phylogenetic distribution of Neisseria gonorrhoeae}

475 isolates. The map shows the countries of isolation of the strains in the collection

476 coloured by continent. The phylogeny shows the relationship among the strains.

477 Coloured strips show (from inside out) the continent of isolation (CONT), year and

478 further typing information (BAPS clusters, NG-MAST, MLST and penA types;

479 colours represent different types or alleles). Mosaic penA types are marked in the

480 outermost black strip.

481 Figure 2 | Global phylogeographic analysis. The dated maximum likelihood

482 phylogenetic tree shows the posterior probabilities for each continent in every node

483 (pie charts). Continents of isolation (prior) are shown as metadata next to the tips. The

484 top left legend contains information on the proportion of strains from different

485 continents before and after the introduction to Asia.

Figure 3 | Evolution of antimicrobial resistance genetic determinants in Neisseria

gonorrhoeae. Antimicrobial resistance determinants (chromosomal mutations and

488 presence/absence of the tetM and blaTEM genes on plasmids (p)) detected in the whole

489 dataset on the maximum likelihood dated tree. Purple represents presence of the

490 determinant and orange its absence. Grey indicates isolates possessing porBla rather

491 than porB $1 b$. The two main lineages are marked as A and B. The left graph shows the

492 proportion of strains with each resistance determinant for both lineages. Statistical

493 significance from a t-test is also shown in the graph. ****p-value $<0.0001$, ***p-

494 value $<0.001,{ }^{*}$ p-value $<0.01,{ }^{*}$ p-value $<0.05$

495 Figure 4 | Characterization of the lineages of Neisseria gonorrhoeae. Proportion of

496 homoplasic sites in all terminal (A) and short terminal branches $(<=100$ SNPs) (B) in

497 lineages A and B and all strains. (C) Distribution of the total number of antimicrobial 
genetic determinants per strains in each lineage. (D) Proportion of strains isolated

500 value $<0.01$

501 Extended Data Figure 1 | Population structure analysis. (a) Discriminant Analysis

502 of Principal Components (DAPC) clustering of the Neisseria gonorrhoeae strains by

503 its continent of isolation (p-value for discriminant function $1<0.001$, Supplementary

504 Table 3) (b) Membership plot showing the posterior probability assignment of each

505 strain to each of the continents. The bar above the plot shows the prior information on

506 the continent of isolation per strain. (c) A-score optimization test performed to obtain

507 the optimal number of principal components to retain in the DAPC analysis ${ }^{36}$ as a

508 trade-off between power of discrimination and over-fitting. Calculated as the

509 difference between the proportion of successful reassignments and values obtained

510 using random groups corrected by the number of retained components

511 (http://adegenet.r-forge.r-project.org/files/tutorial-dapc.pdf). (d) DAPC analysis with

512 randomized continents showing no population differentiation by continent.

513 Extended Data Figure 2 | Temporal signal, tMRCA and substitution rate

514 estimation. (a) Root-to-tip distance estimate over time of the $419 \mathrm{~N}$. gonorrhoeae

515 global collection (first plot) and the different BAPS clusters (following plots)

516 calculated using the "clustered permutation" approach developed by Murray et al,

$5172017^{37}$. (b) Comparison between LSD $^{38}$ and BEAST ${ }^{39}$. Estimates of the tMRCA and

518 the substitution rate for the five BAPS clusters that reached convergence in BEAST as

519 well as the whole collection calculated using BEAST and LSD. (c) Distribution of the

520 tree root height and substitution rate parameters estimated from two different BEAST

521 chains after 100 million generations (burn-in = 30 million). The 95\% HPD interval 
522 from BEAST and the confidence interval obtained with LSD are plotted below for

523 comparison.

524 Extended Data Figure 3 | Phylogeographic analysis. (a) Maximum likelihood

525 phylogenetic tree showing the tips coloured with the prior information on the

526 continent of isolation (inner circle) and the posterior group membership after DAPC

527 analysis $^{36}$ (outer circle). Admixed individuals are defined as those with $<80 \%$

528 posterior probability of assignment to any of the five continents and are shown in

529 grey. Light blue circles represent bootstrap support values above 70\%. (b) Stochastic

530 mapping of the posterior continent assignments over the dated phylogenetic tree

531 complemented with US strains from Grad et al. $2014^{15}$ and using even number of

532 strains from each continent $(\mathrm{N}=41)$ except for Oceania, for which there is not more

533 available data in the public databases at the time of writing. Analysis was performed

534 using the phytools $\mathrm{R}$ package $\mathrm{e}^{40}$. Pie charts in every node represent the proportion of

535 ancestry to each of the five continents. (c-d) Per-branch posterior distribution of the

536 trait "Continent" using both prior (c) and posterior (d) memberships along the

537 phylogenetic tree calculated using treeBreaker ${ }^{41}$. (e-f) Distribution of isolation dates

538 by continent shown for both prior (e) and posterior (f) continent assignments. The

539 width of the boxplots is proportional to the number of strains from each continent.

540 Extended Data Figure 4 | Minimum Inhibitory Concentration (MIC, in mg/L)

541 for six antimicrobials. The distribution of the MICs of six antimicrobials is shown

542 for all strains included in the analysis in $\log 2$ scale. Note that the distribution of

543 resistance is equivalent to that found from genotypic resistance. The scale of the

544 cephalosporins CFX and CRO has been collided into one and the range of MICs in

545 the legend is shown without logging. The minimum MIC value has been set to 0.016 
$546 \mathrm{mg} / \mathrm{L}$ for all antimicrobials. TET $=$ Tetracycline, $\mathrm{PEN}=$ Penicillin $\mathrm{G}, \mathrm{CIP}=$

547 Ciprofloxacin, $\mathrm{CFX}=$ Cefixime, $\mathrm{CRO}=$ Ceftriaxone, $\mathrm{AZM}=$ Azithromycin.

548 Extended Data Figure 5 | Phenotypic and genotypic resistance. Distribution of

549 MIC values (in logarithmic scale) for each combination of antimicrobial determinants

550 associated in the literature ${ }^{42}$ to each of the six antimicrobials under study. Horizontal

551 dashed lines mark EUCAST breakpoints (www.eucast.org) except for azithromycin,

552 in which the CLSI $2 \mu \mathrm{g} / \mathrm{mL}$ upper bound is used (www.clsi.org).

553 Extended Data Figure 6 | Most predicted acquisitions of antimicrobial resistance

554 happened after the introduction of antimicrobials. The distribution of the predicted

555 acquisitions of the known determinants of antimicrobial resistance along time are

556 represented as half violin plots (pink when using the date of the anterior and blue the

557 posterior nodes of the branch where the acquisition is predicted). The prediction was

558 performed with ancestral maximum likelihood reconstruction (ace function of the

559 phytools $\mathrm{R}$ package ${ }^{43}$ ) and a symmetric model of transition between states. Black

560 diamonds mark the reported date of introduction of different antimicrobials to treat

561 gonorrhoea and orange diamonds the first reported date of treatment failure. The

562 barplot on the right shows the total number of predicted acquisitions for each

563 mutation, and it is coloured to differentiate mutations affecting different

564 antimicrobials.

565 Extended Data Figure 7 | Distribution of the Gonococcal Genomic Island and

566 plasmids. Maximum likelihood phylogenetic tree showing, from the inner to the outer

567 circle: the occurrence of the Gonococcal Genomic Island (GGI) and the three main

568 plasmids (pCryptic-P1, pConjugative-P2 and pBlaTEM-P3). Colours in plasmid

569 tracks correspond to different types (see legend). The two lineages are marked in 
black and purple, respectively. Node shapes represent bootstrap support values above

$57170 \%$.

\section{Extended Data Figure 8 | AMR genetic determinants in the two lineages. (a)}

573 Mean number of changes from susceptible to antimicrobial resistant status inferred to

574 have evolutionarily happened in lineages A and B for all the analysed resistance

575 determinants, including the penA mosaic. Values have been corrected by the number

576 of edges of the $\mathrm{A}(\mathrm{N}=586)$ and $\mathrm{B}(\mathrm{N}=236)$ subtree lineages, respectively. (b)

577 Distribution of the patient's gender and the number of antimicrobial resistance

578 determinants that the infecting strains carry. The figure shows a non-recombinant

579 maximum likelihood tree of 639 strains (263 from the global collection and 376 from

580 two North American studies ${ }^{16,25}$ ) with the mentioned information as metadata.

581 Lineages A and B are also labelled.

582 Extended Data Figure 9 | Assessment of recombination SNPs and homoplasic

583 sites in terminal branches. (a) Proportion of SNPs inside the recombination events

584 predicted by Gubbins for all terminal branches together and shorter $(<=100$ SNPs $)$

585 and longer (>100 SNPs) terminal branches separately in lineages A and B and all

586 strains. (b) Proportion of homoplasic sites in all terminal branches together and

587 shorter (<=100 SNPs) and longer (>100 SNPs) terminal branches separately lineages

588 A, B and all strains. (c) Distribution of the p-values calculated using a Student's t test

589 on the number of homoplasies in the two lineages on short and long terminal branches

590 of the tree at different SNP cut-offs. Significance thresholds at 0.01 and 0.05 are

591 marked with a dashed line. Short branches are probably more reliable for this type of

592 calculation than long branches and this can be observed at around a cut-off of 100

593 SNPs, where branches under this number of SNPs have a p-value $<0.005$ while the

594 rest are clearly not significant $\mathrm{p}$-value>0.1. SNPs in repeat regions and those known 
to undergo antigenic variation, such as pilin-, opA54- or Maf-associated genes were

excluded from the calculation. ${ }^{*}$ pvalue $<0.01,{ }^{*}$-value $<0.05, \mathrm{~ns}=$ non-significant.

598 from the Euro-GASP 2013 survey $^{\mathbf{2 6}}$. The two lineages (A in purple and B in black)

599 were identified by combining this set with the global collection data as specified in

600 the methods section. The metadata aligned to the tree shows the distribution of the

601 gender of the patients from which the isolates were obtained, the type of transmission

602 and the SIR categories of the phenotypic antimicrobial susceptibility test of the

603 isolates following the breakpoints from EUCAST (www.eucast.org). Counts of each

604 column are shown per lineage on the right side. Asterisks in lineage B indicate

605 statistical significance compared to lineage A. AZM $=$ Azithromycin, $\mathrm{CFX}=$

606 Cefixime, $\mathrm{CIP}=$ Ciprofloxacin. $\mathrm{R}=$ Resistant, $\mathrm{I}=$ Intermediate resistance, $\mathrm{S}=$

607 Susceptible. $* * * *$ p-value $<0.0001, * *$ p-value $<0.01$

608 Supplementary Table 1 | Additional strain information. Metadata associated to the

$609419 N$. gonorrhoeae strains included in the study, typing information and

610 antimicrobial resistance determinants detected by ARIBA.

611 Supplementary Table 2 | Analysis of Molecular Variance. Analysis of Molecular

612 Variance (AMOVA) calculated using three population levels: continent, subcontinent

613 and country, for real and randomized population structure. Randomization shows no

614 population structure at all, supporting the signal obtained by our data.

615 Supplementary Table 3 | Multivariate Analysis of Variance. Results of the

616 Multivariate Analysis of Variance (MANOVA, Wilk's lambda test) calculated to

617 assess the significance of each discriminant function in the DAPC analysis. ****p-

618 value $<0.0001$ 
619 Supplementary Table 4 | Prior and posterior continent assignments. Prior and

620 posterior assignments to continents for each of the strains included in the study after

621 running a DAPC analysis.

622 Supplementary Table 5 | Membership assignments by continent. Number of prior

623 and posterior membership assignments to each continent after DAPC analysis.

624 Supplementary Table 6 | Root ancestry. Percentage of ancestry of the root node of

625 the tree to each of the five continents (posterior assignments). Summary of 1000

626 stochastic maps. The analysis was repeated by incorporating extra American strains

627 from Grad et al $2014^{15}$. The number of strains with a posterior assignment to each

628 continent used in each analysis is specified between brackets. Even sampling was

629 obtained by subsampling the strains from different continents 100 times and

630 performing 10 stochastic maps on each of them. Continents were randomised on an

631 evenly sampled tree including extra American strains and the analysis repeated using

6321000 stochastic maps. The highest percentage is highlighted.

633 Supplementary Table 7 | Estimates of the substitution rate and tMRCA from

634 BEAST and LSD. Estimates of substitution rate and time for the Most Recent

635 Common Ancestor (tMRCA) for five BAPS clusters obtained with BEAST and LSD.

$636 \mathrm{R}^{2}$ shows the correlation coefficient between the root-to-tip distances and dates of

637 isolation. HPD = High Posterior Density. 


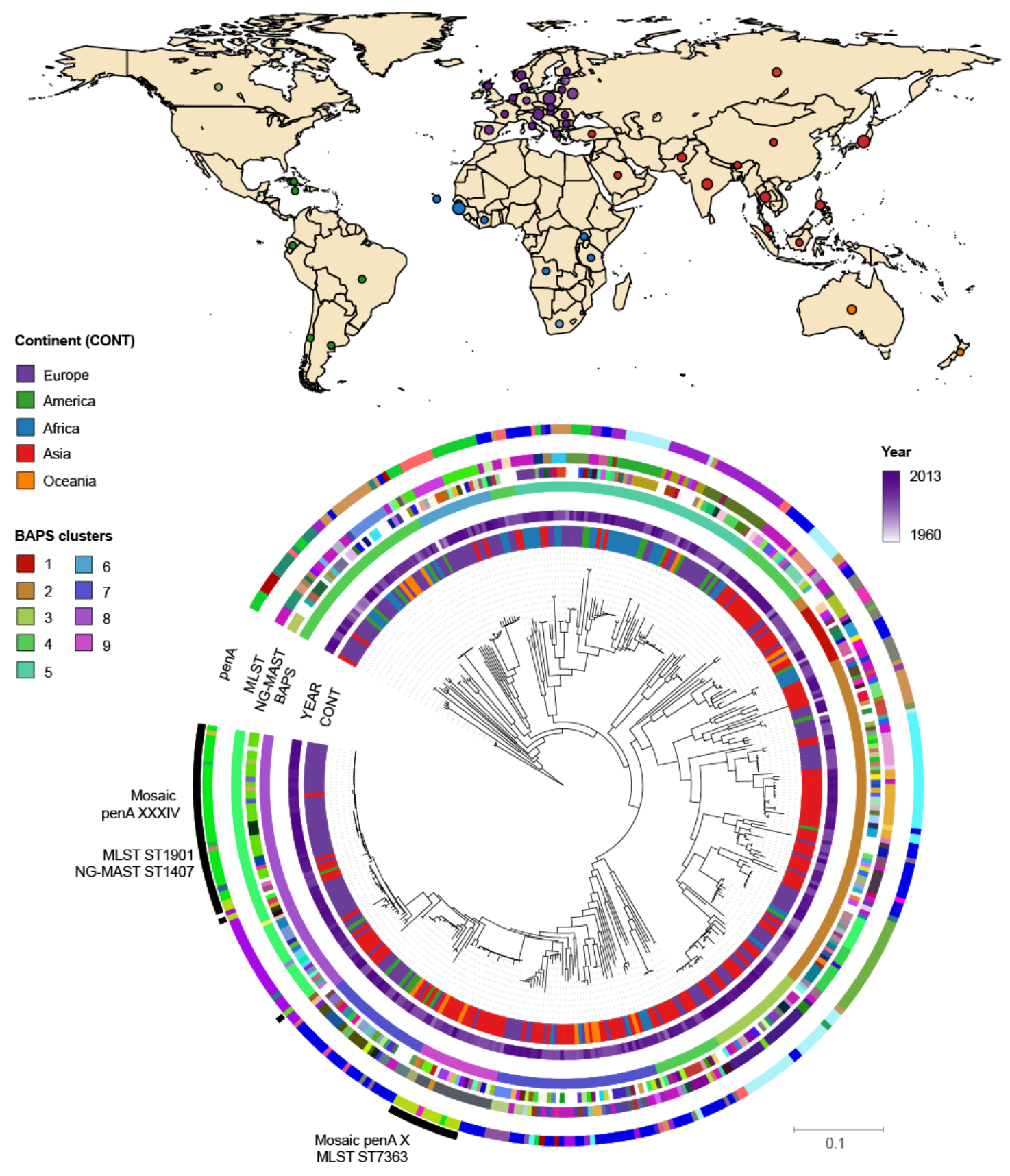

Figure 1 | Geographic and phylogenetic distribution of Neisseria gonorrhoeae

isolates. The map shows the countries of isolation of the strains in the collection coloured by continent. The phylogeny shows the relationship among the strains.

Coloured strips show (from inside out) the continent of isolation (CONT), year and further typing information (BAPS clusters, NG-MAST, MLST and penA types; colours represent different types or alleles). Mosaic penA types are marked in the outermost black strip. 

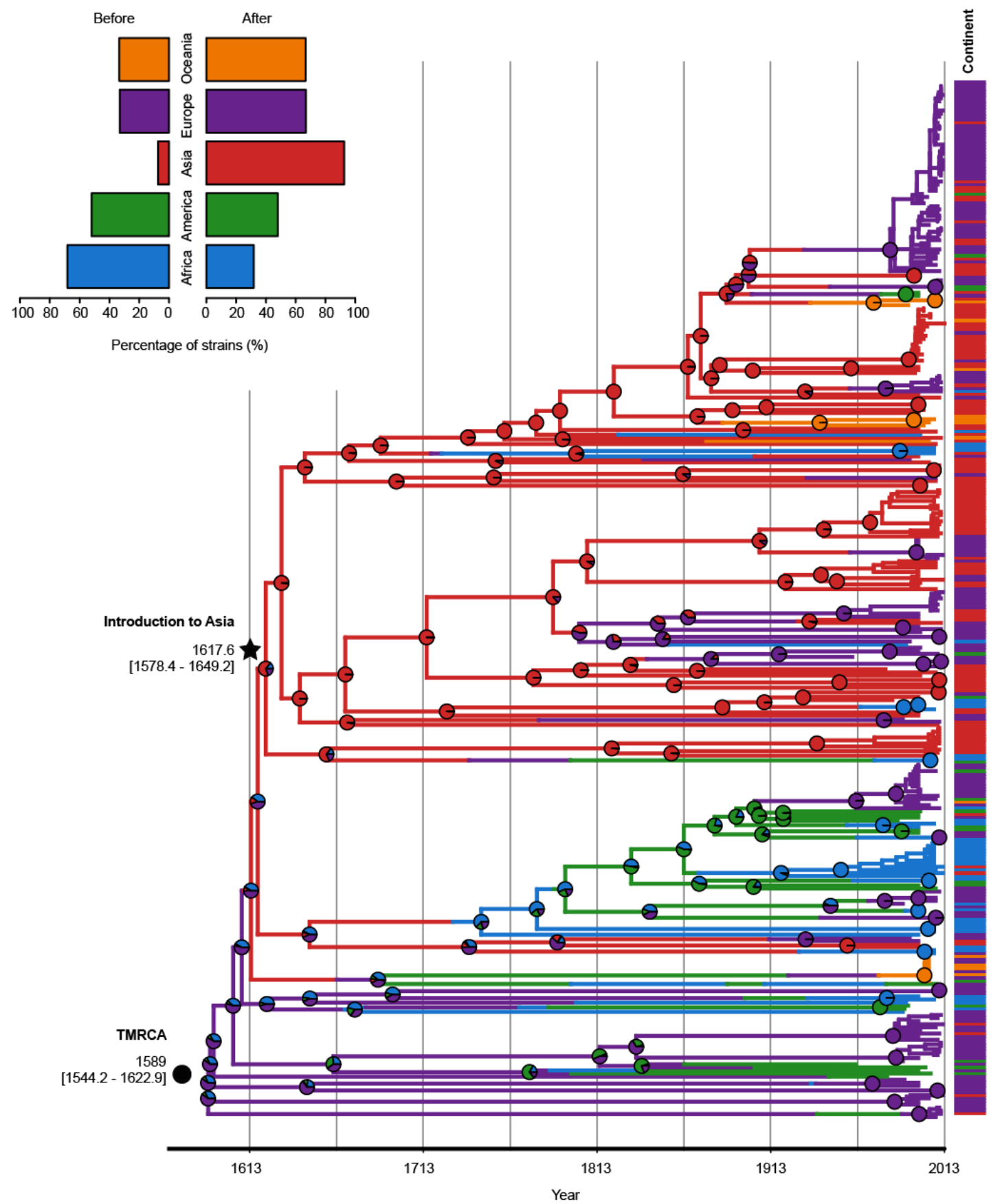

Figure 2 | Global phylogeographic analysis. The dated maximum likelihood

phylogenetic tree shows the posterior probabilities for each continent in every node

(pie charts). Continents of isolation (prior) are shown as metadata next to the tips. The top left legend contains information on the proportion of strains from different continents before and after the introduction to Asia. 


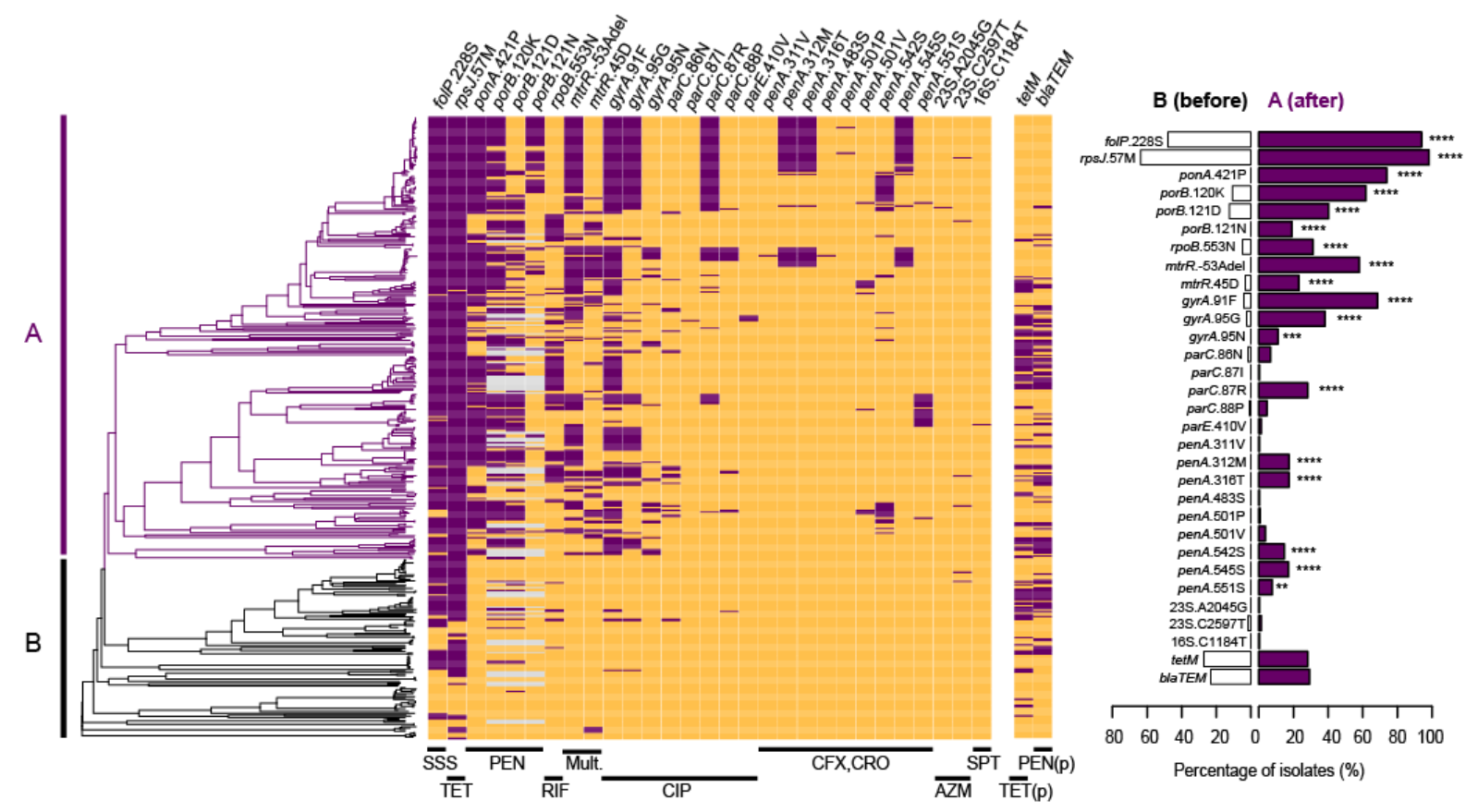

Figure 3. Evolution of antimicrobial resistance genetic determinants in Neisseria

gonorrhoeae. Antimicrobial resistance determinants (chromosomal mutations and presence/absence of the tetM and blaTEM genes on plasmids (p)) detected in the whole dataset on the maximum likelihood dated tree. Purple represents presence of the determinant and orange its absence. Grey indicates isolates possessing porBla rather than porB1b. The two main lineages are marked as lineage A and B. The left graph shows the proportion of strains with each resistance determinant for both lineages. Statistical significance from a t-test is also shown in the graph. $* * * * \mathrm{p}$-value $<0.0001, * * * \mathrm{p}$-value $<0.001, * * \mathrm{p}$-value $<0.01,{ }^{*} \mathrm{p}$ value $<0.05$. 
a

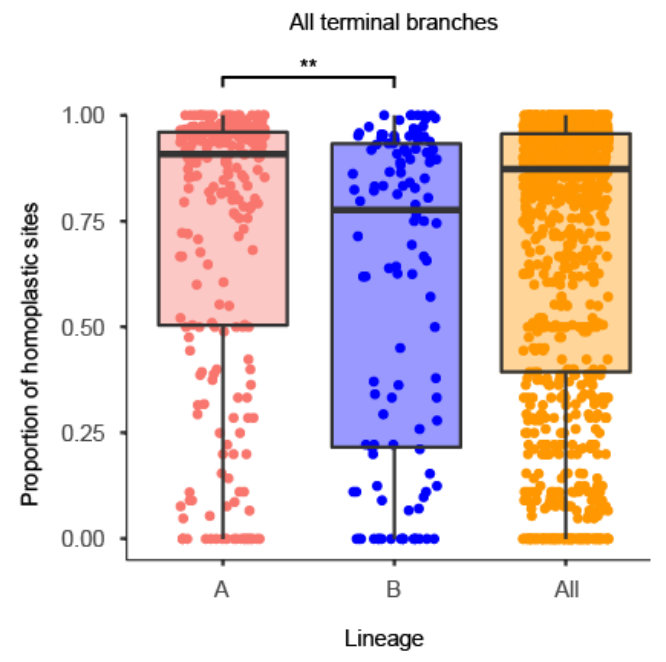

c

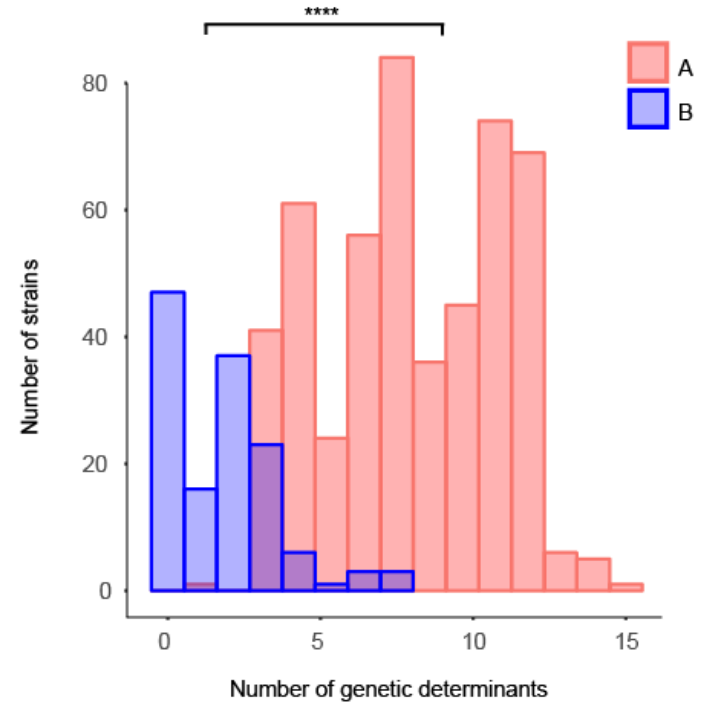

b Short terminal branches (<=100 SNPs)

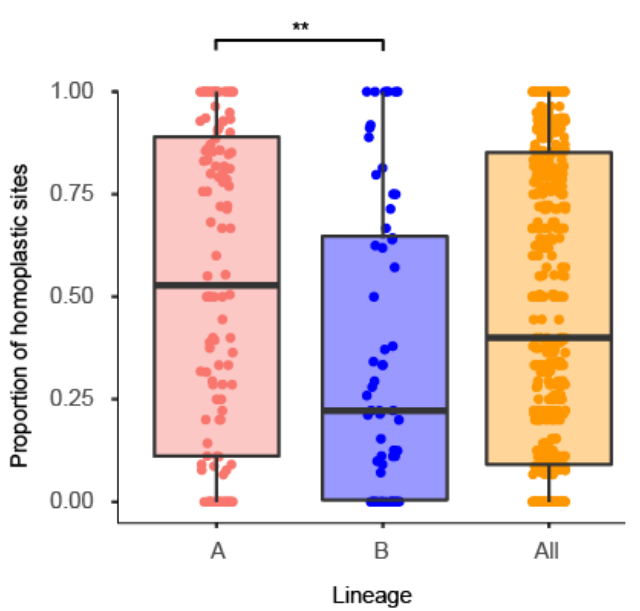

d

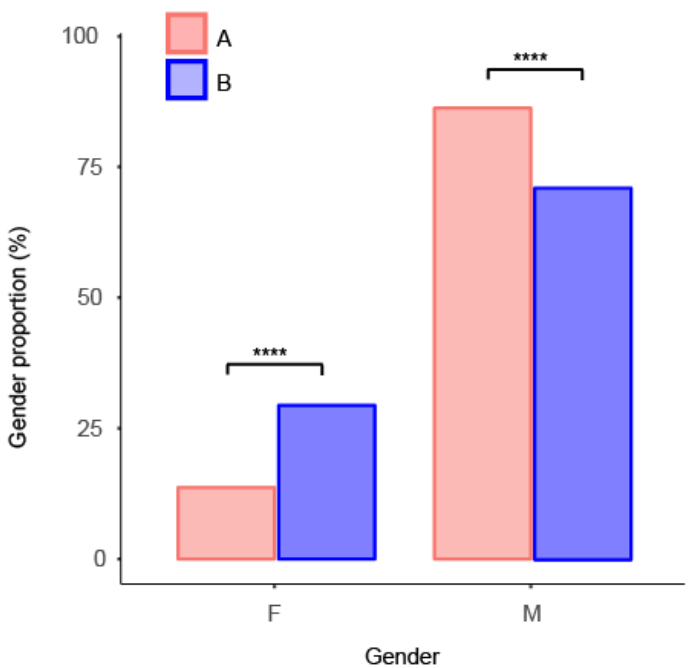

Figure 4. Characterization of the lineages of Neisseria gonorrhoeae. Proportion of homoplasic sites in all terminal (A) and short terminal branches $(<=100$ SNPs) (B) in lineages A and B and all strains. (C) Distribution of the total number of antimicrobial genetic determinants per strains in each lineage. (D) Proportion of strains isolated from female (F) and male (M) patients in each lineage. $* * * *$ p-value $<0.0001, * *$ p-value $<0.01$. 


\section{Methods}

2 Global $N$. gonorrhoeae strains and antimicrobial susceptibility testing

3 A total of $413 N$. gonorrhoeae strains without known epidemiological relatedness were

4 collected from patients suffering gonorrhoea in 58 countries spanning five continents

5 (Supplementary Table 1). Six genome references were also included in the study, spanning a

6 range of isolation dates between 1960 and 2013 in total. Bacterial isolation from the

7 corresponding samples, preservation and transportation was performed following standard

8 microbiological procedures ${ }^{44}$. $\beta$-lactamase production and minimum inhibitory concentrations

9 (MIC) were tested for a range of antimicrobials as described previously ${ }^{45}$ : spectinomycin,

10 tetracycline, penicillin G, ciprofloxacin, azithromycin, cefixime and ceftriaxone.

\section{DNA preparation and whole-genome sequencing}

12 All isolates were confirmed to be $N$. gonorrhoeae and genomic DNA was extracted from the 13 isolates using the Promega Wizard DNA purification kit, following the instructions from the 14 manufacturer. Purified DNAs were multiplexed and sequenced using two lanes of the HiSeq $1525002 \times 100$ bp platform at the Wellcome Sanger Institute.

\section{Mapping and variant calling}

17 Fastq files from the 413 new gonococcal strains and the N. meningitidis 10356_1\#65 outgroup 18 (ENA accession ERS248641) were mapped to a common reference, $N$. gonorrhoeae FA1090 19 (NCBI accession NC_002946, 2,153,922 bp) using $\quad$ SMALT v0.7.4

20 (http://www.sanger.ac.uk/science/tools/smalt-0). Variants were called using SAMtools and 21 BCFtools v1.2 $2^{46}$ after indel realignment with GATK v1.5.9 $9^{47}$ and further filtered as described 22 previously ${ }^{48}$

23 Six public reference genomes were obtained from the NCBI and aligned using 24 progressiveMAUVE v2.3.149. The XMFA output alignment was converted into a plain fasta 
bioRxiv preprint doi: https://doi.org/10.1101/334847; this version posted May 31, 2018. The copyright holder for this preprint (which was not certified by peer review) is the author/funder, who has granted bioRxiv a license to display the preprint in perpetuity. It is made available under aCC-BY-NC-ND 4.0 International license.

25 format using $N$. gonorrhoeae FA1090 as reordering reference through a custom Perl script.

26 Positions with gaps in this reference were removed, so that the resulting alignment had

27 homologous positions to the 2,153,922 bp in the FA1090 genome. This alignment was added 28 into the one resulting from mapping the 413 isolates, producing a 419 -strains alignment, which 29 was used for further analyses.

\section{Recombination removal and phylogenetic reconstruction}

31 Phages described in the $N$. gonorrhoeae FA1090 strain ${ }^{50}$ were masked in the alignment before running Gubbins ${ }^{51}$, which was used to identify genome-wide recombinant segments using high

33 single nucleotide polymorphism (SNP) density as a marker. The N. meningitidis 10356_1\#65 strain was used as outgroup so that events affecting all $N$. gonorrhoeae strains were not excluded from subsequent calculations.

The detected recombination events and repeat regions inferred by repeat-match (MUMMER v3.23) ${ }^{52}$ on the $N$. gonorrhoeae FA1090 strain genome were masked in order to minimize the occurrence of false-positive SNPs. Gblocks v0.91b ${ }^{53}$ was run on the resulting alignment to further clean poorly aligned regions that may introduce noise to phylogenetic analyses. Gblocks was run by allowing gap positions in up to $50 \%$ of the sequences, with a minimum

41 block length of 10 and 8 as maximum number of contiguous non-conserved positions. The resulting 1,211,180 bp clean alignment included 15,562 variable sites, identified by snp_sites ${ }^{54}$, and was used for population structure analysis, phylogenetic inference and

44 divergence estimation. Genetic clusters were obtained from the non-recombining alignment using hierBAPS 55 .

The final SNP alignment was used for Maximum Likelihood (ML) phylogenetic tree 47 reconstruction using RAxML v7.8.6 $6^{56}$ under the GTRGAMMA model of nucleotide substitution and 100 bootstrap replicates. Ancestral states of all SNPs before recombination 
bioRxiv preprint doi: https://doi.org/10.1101/334847; this version posted May 31, 2018. The copyright holder for this preprint (which was

not certified by peer review) is the author/funder, who has granted bioRxiv a license to display the preprint in perpetuity. It is made available under aCC-BY-NC-ND 4.0 International license.

removal were reconstructed onto the resulting phylogenetic tree using ACCTRAN transformation in python. Homoplasic sites in the terminal branches of the tree were detected

51 and evaluated for the two main lineages.

\section{Genome de novo assembly and in silico typing}

53 In parallel to the mapping process, reads were assembled using the assembly and improvement

54 iterative pipeline developed at the Wellcome Sanger Institute ${ }^{57}$. Multi-locus sequence typing $55 \quad(\mathrm{MLST})^{58}$ and $N$. gonorrhoeae multi-antigen sequence typing (NG-MAST) ${ }^{59}$ typing schemes were retrieved directly from the sequences using the get_sequence_type script (https://github.com/sanger-pathogens/mlst_check/blob/master/bin/get_sequence_type) and NGMASTER ${ }^{60}$, respectively. The presence of the $\beta$-lactamase (blaTEM) and tetracycline (tet $M$ ) genes on plasmids and the Gonococcal Genomic Island (GGI) were detected using BLAST v2.3.0+ ${ }^{61}$ and ARIBA v2. ${ }^{62}$. Typing was performed for the conjugative plasmid and the

61 blaTEM plasmids using an in-silico PCR (https://github.com/simonrharris/in_silico_pcr).

62 Primers to differentiate between the Dutch and the American tetM-containing plasmids were obtained from Turner et al, $1999^{63}$. To type the blaTEM plasmids, primers described in Dillon et al, $1999^{64}$ were used and the resulting amplicon sizes evaluated to differentiate among the Asia, Africa and Toronto/Rio types (Supplementary Table 1).

\section{Analysis of population structure}

67 In order to study population structure from the resulting alignment, the poppr $\mathrm{R}$ package ${ }^{65}$ was used to perform an AMOVA test on the non-recombining section of the genome ${ }^{66}$ on three

69 geographical hierarchies: continent, subcontinent and country, to calculate the percentage of 70 observed variance within and between groups. In order to test if the observed differentiation

71 between continents was significant, a randomization test $(\mathrm{N}=1,000$ permutations $)$ was 
performed using the randtest function from the ade $4 \mathrm{R}$ package ${ }^{67}$, which randomly permutes the population structure to assess the observed signal of differentiation.

74 To further study population structure, a Discriminant Analysis of Principal Components (DAPC, adegenet $\mathrm{R}$ package) $)^{36,68}$ analysis was applied to the non-recombining 15,562 SNPs alignment using continent of isolation as population. The procedure followed by this multivariate discriminant analysis tries to maximize the discrimination between the predefined groups. To avoid over-fitting and keep enough discrimination power, the optimal number of principal components (PC) to retain was determined using the a-score optimization test, which uses randomized groups to calculate the proportion of successful reassignments corrected by the number of retained PCs. This methodology resulted in 83 principal components as optimal to keep a balance between discrimination power and over-fitting. Prior assignment to continents was randomized and the DAPC analysis repeated to confirm that the observed separation among clusters does not occur by chance. Four discriminant functions were kept for the analysis, considering the number of variables was 5 continents. A Multivariate Analysis of Variance (MANOVA) test ${ }^{69}$ was applied to test whether there were differences between the means of the different clusters (continents) on the discriminant clustering. Wilks' lambda was used to test the significance of this MANOVA test. Resulting p-values were adjusted for multiple tests using False Discovery Rate (FDR) ${ }^{70}$.

DAPC derives group membership probabilities from the retained discriminant functions.

91 These results were used to evaluate the level of admixture in the dataset under study. Isolates assigned with $>80 \%$ posterior probability to a continent different from the prior assignment

93 were interpreted as intercontinental transmission cases. Isolates with $<80 \%$ of posterior assignment to any of the continents were considered as admixed. 


\section{Divergence estimation with LSD and BEAST}

Year of isolation for all the strains was used to calculate a root-to-tip distance regression versus time to make an estimate of the temporal signal in the data. To do this, a "clustered permutation" approach was used as described ${ }^{37,71}$, which takes into account potential confounding temporal and genetic structure in the data. A total of 1,000 permutations were performed with this method by randomizing the isolation dates in order to get an estimate of the statistical significance of the results. This procedure was applied to the whole dataset and to the different BAPS clusters.

In order to get an estimate of the substitution rate and the Most Recent Common Ancestor (tMRCA) for the whole $N$. gonorrhoeae global collection, the Least-Square Dating (LSD) v0.3 software $^{38}$ was used. This new approach has been shown to be robust to uncorrelated changes of the molecular clock and to give similar results to BEAST ${ }^{38}$. In order to compare the performance between LSD and BEAST, individual BAPS clusters were used. Specifically, Bayesian approximation using BEAST v1.8.2 $2^{39}$ was run to estimate the tMRCA and the substitution rate of the genetic clusters determined by hierBAPS ${ }^{55}$. Three chains were run per cluster up to 100 million generations by using a GTRGAMMA model of nucleotide substitution with 4 categories, strict molecular clock with a diffuse gamma distribution (shape 0.001 and scale 1,000$)$ and a constant population size as priors. The same configuration was used to run two different chains with the whole collection, which did not reach proper convergence because of the complexity of the dataset. LSD was also run for the BAPS clusters that reached convergence in BEAST and the results compared (Supplementary Note 1). The obtained tMRCA was further confirmed using the Wald statistic (Supplementary Note 2). 


\section{Phylogeography with stochastic character mapping}

121 The continent of isolation was used as a discrete trait to study changes in the distribution over

122 the phylogenetic tree using treeBreaker ${ }^{41}$. This program calculates the per-branch posterior

123 probability of having a change in the distribution of a discrete character.

124 Stochastic character mapping ${ }^{72}$ with a symmetric transition model (SYM) was applied to the 125 phylogenetic tree to get posterior probabilities for each continent at every node using the make.simmap function implemented in the phytools $\mathrm{R}$ package ${ }^{40}$. Given a phylogeny and a set

127 of tip states ("continent" in this study), this method uses an MCMC approach to sample 128 character histories from their posterior probability distribution consistent with those states 129 given a model of evolution for the mapped character ${ }^{73}$. This procedure was applied to the prior 130 and posterior continent assignments excluding the admixed individuals to reduce noise from 131 the prior metadata.

132 An extra set of 236 isolates from the $\mathrm{US}^{15}$ was added to the global collection and the 133 phylogeography analyses repeated to confirm our results. To avoid biases due to different 134 number of strains from different continents, the combined datasets were down sampled 100 135 times to $\mathrm{N}=41$ (the maximum number of strains with a posterior assignment to the continent 136 with the least number of strains, Africa), except for Oceania, from which there is not more 137 data in the public databases to include, generating 100 subtrees. Ten stochastic maps were 138 inferred for each of those subtrees and posteriorly combined using phytools ${ }^{40}$, resulting in a 139 total of 1000 evaluated maps.

\section{Evolution of antimicrobial resistance determinants}

141 Mutations conferring antimicrobial resistance in known genetic determinants (16S rRNA, $23 S$ 142 rRNA, rpoB, rpsJ, mtrR, folP, gyrA, parC, parE, penA, ponA and porB) as well as the presence 143 of the $\beta$-lactamase (bla TEM) and tet $M$ genes $^{42}$ were obtained for the 413 strains sequenced in 
144 this study using ARIBA v2.4 $4^{62}$ (Supplementary Table 1) with a custom database created for

$145 N$. gonorrhoeae (precomputed version available in https://github.com/martinghunt/ariba-

146 publication/tree/master/N gonorrhoeae/Ref). Subsequent analyses were performed using $\mathrm{R}^{74}$ :

147 the occurrence of different antimicrobial resistance determinants before and after the change

148 point detected by treeBreaker on the distribution of continents and the distribution of MIC

149 values for penicillin $\mathrm{G}$, tetracycline, ciprofloxacin, ceftriaxone, cefixime and azithromycin

150 against different combinations of the genetic determinants. The average number of changes

151 from a susceptible to a resistant state was inferred for each of the resistant determinants under

152 study in both lineages A and B independently using stochastic mapping (100 simulations) with

153 the make.simmap function implemented in the phytools $\mathrm{R}$ packag $\mathrm{e}^{40}$. The inferred number was

154 corrected by the number of edges in each lineage: $\mathrm{N}=586$ in lineage $\mathrm{A}$ and $\mathrm{N}=236$ in lineage

155 B

156 As an approximation of studying the risk groups characterizing the defined A and B lineages,

157263 isolates from the global collection with information on the gender of the patient were 158 combined with 376 from two North American studies with this information available ${ }^{16,25}$.

159 ARIBA v2.4 was run for the extra isolates and the obtained results joined with the ones from 160 the global dataset. Metadata on gender and number of total resistance determinants detected 161 per strain was plotted on a recombination-free phylogenetic tree obtained as described above 162 and differences between the two lineages evaluated using a t-test with $\mathrm{R}^{74}$.

163 In order to confirm our hypothesis on the two lineages being associated to different risk 164 groups and antimicrobial susceptibilities, we downloaded the phylogenetic tree of 1,054

165 European isolates from a 2013 Euro-GASP survey from the WGSA N. gonorrhoeae 166 scheme ${ }^{26}$ (https://www.wgsa.net/eurogasp2013). The breakpoint between lineages A and B

167 was detected by obtaining a combined core genome alignment of this and our global set 168 (1,473 strains in total) using Roary v3.11.375 and running a pseudo-maximum likelihood tree 
169 with the resulting $\operatorname{SNPs}^{54}$ with FastTree v2.1.976.

\section{$170 \quad$ Visualization}

171 Visualization of metadata in phylogenetic trees was performed using iTOL $^{77}$. Mapping of the

172 and presence/absence of antimicrobial resistance determinants detected with ARIBA were

173 visualized using Phandango ${ }^{78}$.

\section{Data availability}

175 All genomic data has been deposited in the European Nucleotide Archive (ENA) under

176 project number PRJEB4024. Accession numbers for the particular strains are indicated in

177 Supplementary Table 1.

17936 Jombart, T., Devillard, S. \& Balloux, F. Discriminant analysis of principal

180 components: a new method for the analysis of genetically structured populations.

181 BMC Genet 11, 94, doi:10.1186/1471-2156-11-94 (2010).

18237 Murray, G. G. et al. The effect of genetic structure on molecular dating and tests for 183 temporal signal. Methods Ecol Evol 7, 80-89, doi:10.1111/2041-210X.12466 (2016).

18438 To, T. H., Jung, M., Lycett, S. \& Gascuel, O. Fast Dating Using Least-Squares

185 Criteria and Algorithms. Syst Biol 65, 82-97, doi:10.1093/sysbio/syv068 (2016).

18639 Drummond, A. J. \& Rambaut, A. BEAST: Bayesian evolutionary analysis by

187 sampling trees. BMC Evol Biol 7, 214, doi:10.1186/1471-2148-7-214 (2007).

$18840 \quad$ Revell, L. J. \& Graham Reynolds, R. A new Bayesian method for fitting evolutionary 189 models to comparative data with intraspecific variation. Evolution 66, 2697-2707, 190 doi:10.1111/j.1558-5646.2012.01645.x (2012). 
19141 Ansari, M. A. \& Didelot, X. Bayesian Inference of the Evolution of a Phenotype Distribution on a Phylogenetic Tree. Genetics 204, 89-98, doi:10.1534/genetics.116.190496 (2016).

19442 Unemo, M. \& Shafer, W. M. Antimicrobial resistance in Neisseria gonorrhoeae in the 21st century: past, evolution, and future. Clin Microbiol Rev 27, 587-613, doi:10.1128/CMR.00010-14 (2014).

Revell, L. J. phytools: an R package for phylogenetic comparative biology (and other things). Methods in Ecology and Evolution 3, 217-223, doi:10.1111/j.2041210X.2011.00169.x (2012).

$20044 \quad$ Unemo, M., Olcen, P., Berglund, T., Albert, J. \& Fredlund, H. Molecular epidemiology of Neisseria gonorrhoeae: sequence analysis of the porB gene confirms presence of two circulating strains. J Clin Microbiol 40, 3741-3749 (2002).

20345 Unemo, M., Fasth, O., Fredlund, H., Limnios, A. \& Tapsall, J. Phenotypic and genetic characterization of the 2008 WHO Neisseria gonorrhoeae reference strain panel intended for global quality assurance and quality control of gonococcal antimicrobial resistance surveillance for public health purposes. J Antimicrob Chemother 63, 1142-1151, doi:10.1093/jac/dkp098 (2009). 2078-2079, doi:10.1093/bioinformatics/btp352 (2009).

21047 McKenna, A. et al. The Genome Analysis Toolkit: a MapReduce framework for analyzing next-generation DNA sequencing data. Genome Res 20, 1297-1303, doi:10.1101/gr.107524.110 (2010).

21348 Harris, S. R. et al. Evolution of MRSA during hospital transmission and intercontinental spread. Science 327, 469-474, doi:10.1126/science.1182395 (2010). 
21549 Darling, A. E., Mau, B. \& Perna, N. T. progressiveMauve: multiple genome

216 alignment with gene gain, loss and rearrangement. PLoS One 5, e11147,

217 doi:10.1371/journal.pone.0011147 (2010).

21850 Piekarowicz, A. et al. Characterization of the dsDNA prophage sequences in the 219 genome of Neisseria gonorrhoeae and visualization of productive bacteriophage.

220 BMC Microbiol 7, 66, doi:10.1186/1471-2180-7-66 (2007).

$22151 \quad$ Croucher, N. J. et al. Rapid phylogenetic analysis of large samples of recombinant

222 bacterial whole genome sequences using Gubbins. Nucleic Acids Res 43, e15, 223 doi:10.1093/nar/gku1196 (2015).

22452 Kurtz, S. et al. Versatile and open software for comparing large genomes. Genome 225 Biol 5, R12, doi:10.1186/gb-2004-5-2-r12 (2004).

22653 Castresana, J. Selection of conserved blocks from multiple alignments for their use in 227 phylogenetic analysis. Mol Biol Evol 17, 540-552 (2000).

22854 Page, A. J. et al. SNP-sites: rapid efficient extraction of SNPs from multi-FASTA alignments. Microbial Genomics 2, doi:http://dx.doi.org/10.1099/mgen.0.000056 (2016).

23155 Cheng, L., Connor, T. R., Siren, J., Aanensen, D. M. \& Corander, J. Hierarchical and spatially explicit clustering of DNA sequences with BAPS software. Mol Biol Evol 30, 1224-1228, doi:10.1093/molbev/mst028 (2013).

23456 Stamatakis, A. RAxML-VI-HPC: maximum likelihood-based phylogenetic analyses 235 with thousands of taxa and mixed models. Bioinformatics 22, 2688-2690, 236 doi:10.1093/bioinformatics/btl446 (2006).

23757 Page, A. J. et al. Robust high-throughput prokaryote de novo assembly and 238 improvement pipeline for Illumina data. Microbial Genomics, doi:10.1099/mgen.0.000083 (2016). 
$24058 \quad$ Jolley, K. A. \& Maiden, M. C. BIGSdb: Scalable analysis of bacterial genome

241 variation at the population level. BMC Bioinformatics 11, 595, doi:10.1186/1471-

$242 \quad 2105-11-595(2010)$

24359 Martin, I. M., Ison, C. A., Aanensen, D. M., Fenton, K. A. \& Spratt, B. G. Rapid 244 sequence-based identification of gonococcal transmission clusters in a large

245 metropolitan area. J Infect Dis 189, 1497-1505, doi:10.1086/383047 (2004).

24660 Kwong, J. C. et al. NGMASTER: in silico Multi-Antigen Sequence Typing for $247 \quad$ Neisseria gonorrhoeae. bioArxiv, doi:10.1101/057760 (2016).

24861 Camacho, C. et al. BLAST+: architecture and applications. BMC Bioinformatics 10, 249 421, doi:10.1186/1471-2105-10-421 (2009).

25062 Hunt, M. et al. ARIBA: rapid antimicrobial resistance genotyping directly from 251 sequencing reads. Microbial Genomics 3, 1-11, doi:10.1099/mgen.0.000131 (2017).

25263 Turner, A., Gough, K. R. \& Leeming, J. P. Molecular epidemiology of tetM genes in 253 Neisseria gonorrhoeae. Sex Transm Infect 75, 60-66 (1999).

25464 Dillon, J. R., Li, H., Yeung, K. \& Aman, T. A. A PCR assay for discriminating $255 \quad$ Neisseria gonorrhoeaebeta-lactamase-producing plasmids. Mol Cell Probes 13, 8992, doi:10.1006/mcpr.1998.0216 (1999).

25765 Kamvar, Z. N., Tabima, J. F. \& Grunwald, N. J. Poppr: an R package for genetic 258 analysis of populations with clonal, partially clonal, and/or sexual reproduction. 259 PeerJ 2, e281, doi:10.7717/peerj.281 (2014).

26066 Excoffier, L., Smouse, P. E. \& Quattro, J. M. Analysis of molecular variance inferred 261 from metric distances among DNA haplotypes: application to human mitochondrial 262 DNA restriction data. Genetics 131, 479-491 (1992).

26367 Dray, S. \& Dufour, A. B. The ade4 package: implementing the duality diagram for 264 ecologists. Journal of Statistical Software. 22, 1-20 (2007). 
26568 Jombart, T. adegenet: a R package for the multivariate analysis of genetic markers. Bioinformatics 24, 1403-1405, doi:10.1093/bioinformatics/btn129 (2008).

26769 Louis, T. A. Multivariate analysis of variance and repeated measures, a practical approach for behavioural scientists. D. J. Hand and C. C. Taylor, Chapman \& Hall,

$27070 \quad$ Benjamini, Y. Discovering the false discovery rate. Journal of the Royal Statistical Society: Series B (Statistical Methodology) 72, 405-416, doi:10.1111/j.14679868.2010.00746.x (2010).

71 Duchene, S., Duchene, D., Holmes, E. C. \& Ho, S. Y. The Performance of the DateRandomization Test in Phylogenetic Analyses of Time-Structured Virus Data. Mol Biol Evol 32, 1895-1906, doi:10.1093/molbev/msv056 (2015). morphological characters. Syst Biol 52, 131-158 (2003). phylogenies. BMC Bioinformatics 7, 88, doi:10.1186/1471-2105-7-88 (2006). Computing. (2008). doi:10.1371/journal.pone.0009490 (2010).

28777 Letunic, I. \& Bork, P. Interactive tree of life (iTOL) v3: an online tool for the display and annotation of phylogenetic and other trees. Nucleic Acids Res, 
bioRxiv preprint doi: https://doi.org/10.1101/334847; this version posted May 31, 2018. The copyright holder for this preprint (which was

not certified by peer review) is the author/funder, who has granted bioRxiv a license to display the preprint in perpetuity. It is made available under aCC-BY-NC-ND 4.0 International license.

29078 Hadfield, J. et al. Phandango: an interactive viewer for bacterial population genomics. Bioinformatics, 1-2, doi: 10.1093/bioinformatics/btx610 (2017). 


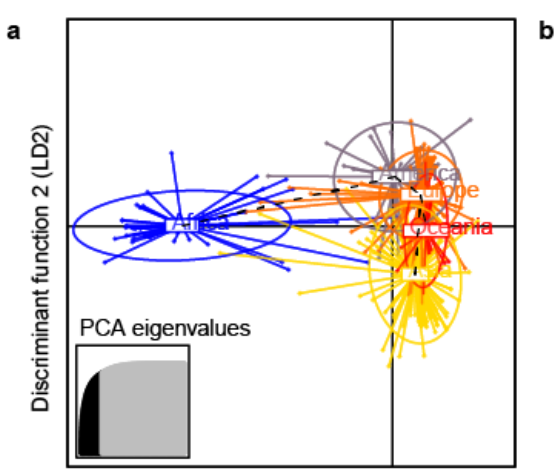

Discriminant function 1 (LD1)

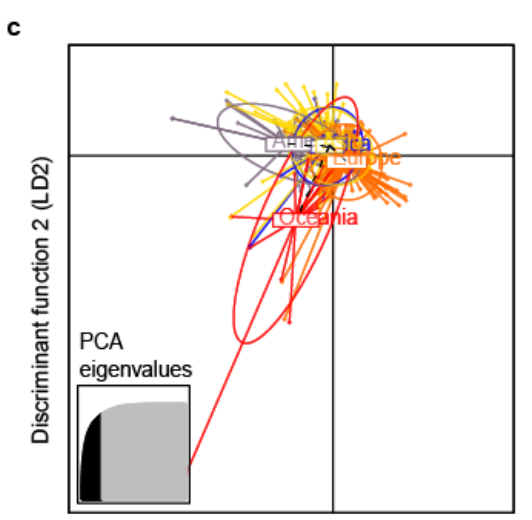

Discriminant function 1 (LD1)

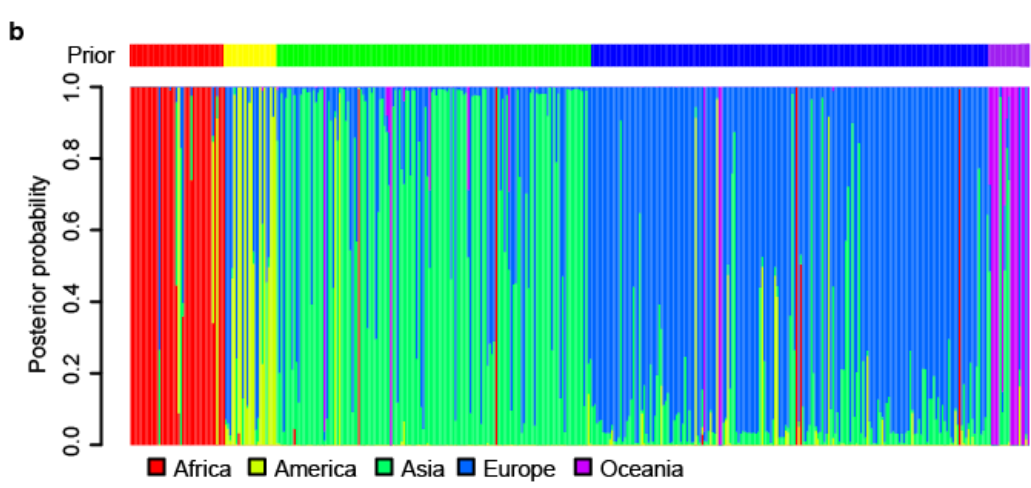

d

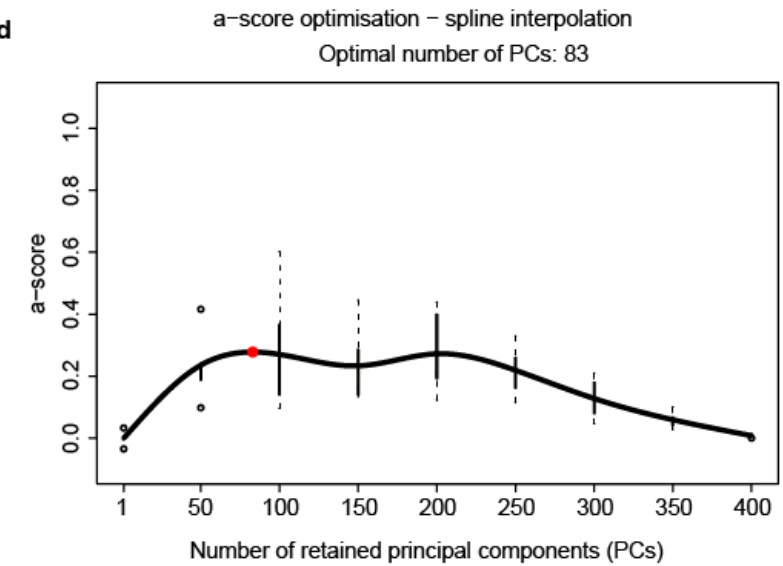

Extended Data Figure 1 | Population structure analysis. (a) Discriminant Analysis of

Principal Components (DAPC) clustering of the Neisseria gonorrhoeae strains by its continent of isolation (p-value for discriminant function $1<0.001$, Supplementary Table 3 ) (b) Membership plot showing the posterior probability assignment of each strain to each of the continents. The bar above the plot shows the prior information on the continent of isolation per strain. (c) A-score optimization test performed to obtain the optimal number of principal components to retain in the DAPC analysis ${ }^{36}$ as a trade-off between power of discrimination and over-fitting. Calculated as the difference between the proportion of successful reassignments and values obtained using random groups corrected by the number of retained components (http://adegenet.r-forge.r-project.org/files/tutorial-dapc.pdf). (d) DAPC analysis with randomized continents showing no population differentiation by continent. 

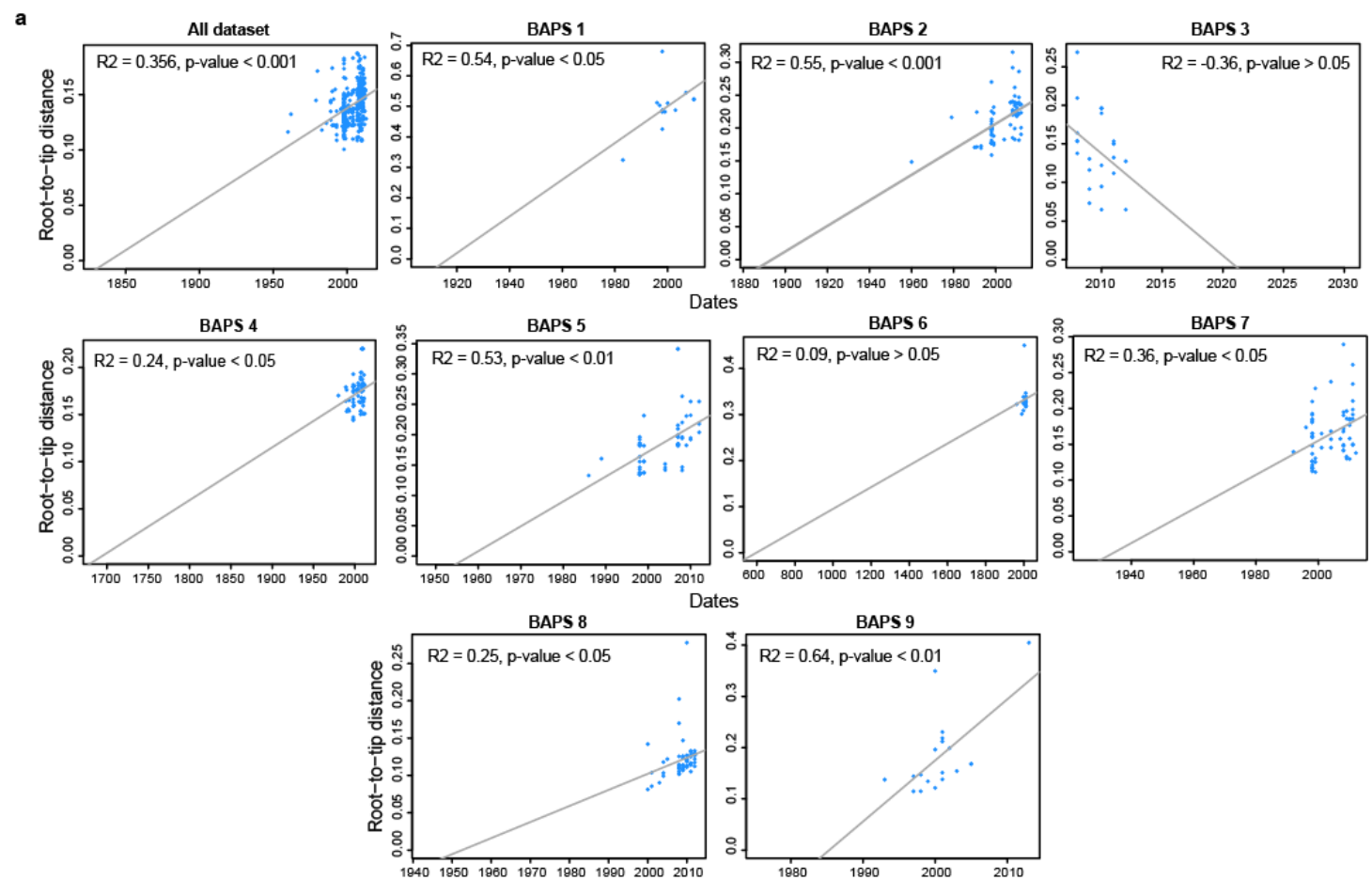

BAPS 9

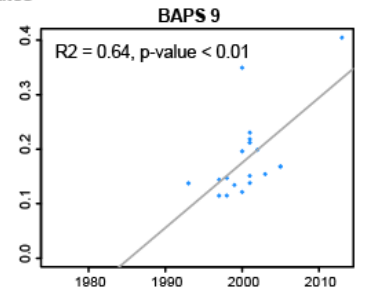

b

Dates

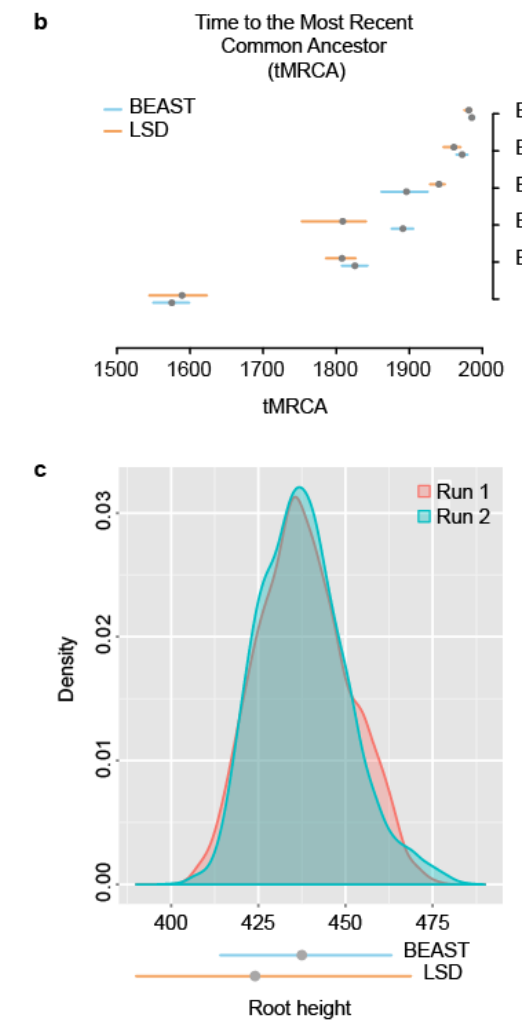

Substitution rate (substitutions/site/year)
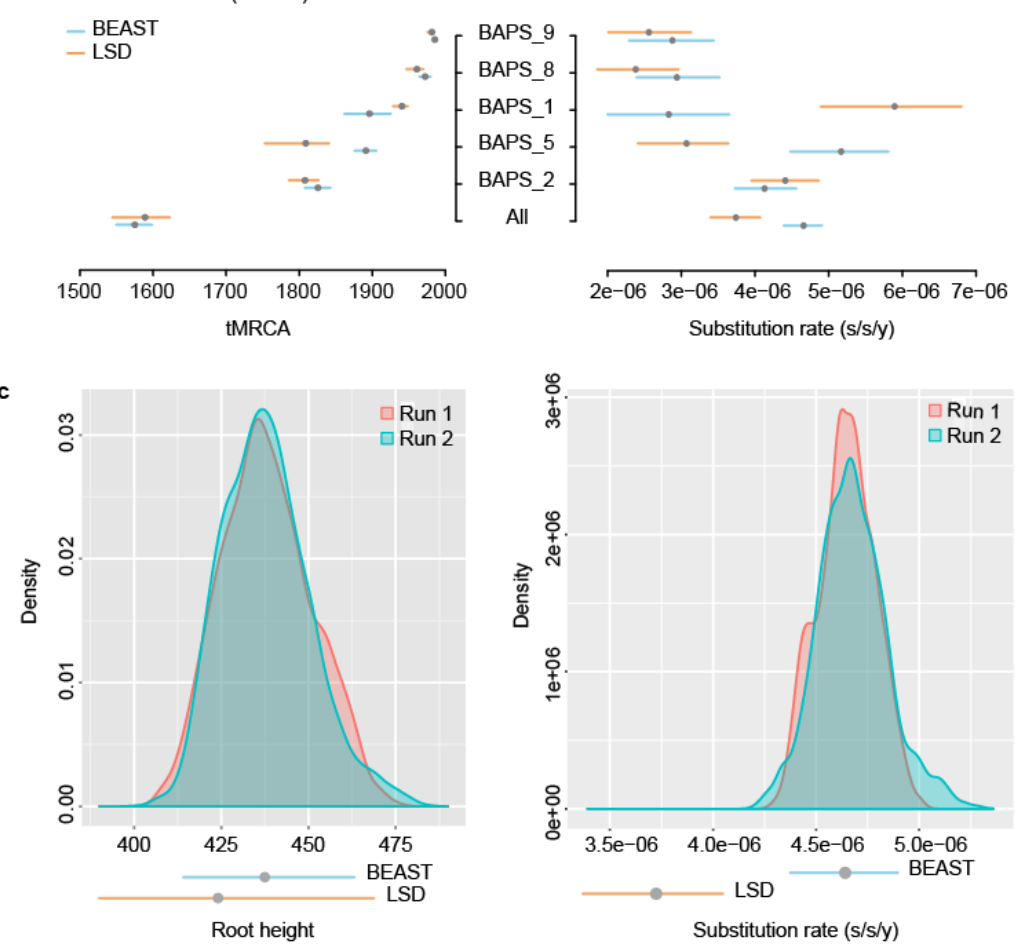

\section{Extended Data Figure 2 | Temporal signal, tMRCA and substitution rate}

estimation. (a) Root-to-tip distance estimate over time of the 419 N. gonorrhoeae global collection (first plot) and the different BAPS clusters (following plots) calculated using the "clustered permutation" approach developed by Murray et al, 
$2017^{37}$. (b) Comparison between $\operatorname{LSD}^{38}$ and BEAST ${ }^{39}$. Estimates of the tMRCA and the substitution rate for the five BAPS clusters that reached convergence in BEAST as well as the whole collection calculated using BEAST and LSD. (c) Distribution of the tree root height and substitution rate parameters estimated from two different BEAST chains after 100 million generations (burn-in = 30 million). The 95\% HPD interval from BEAST and the confidence interval obtained with LSD are plotted below for comparison. 

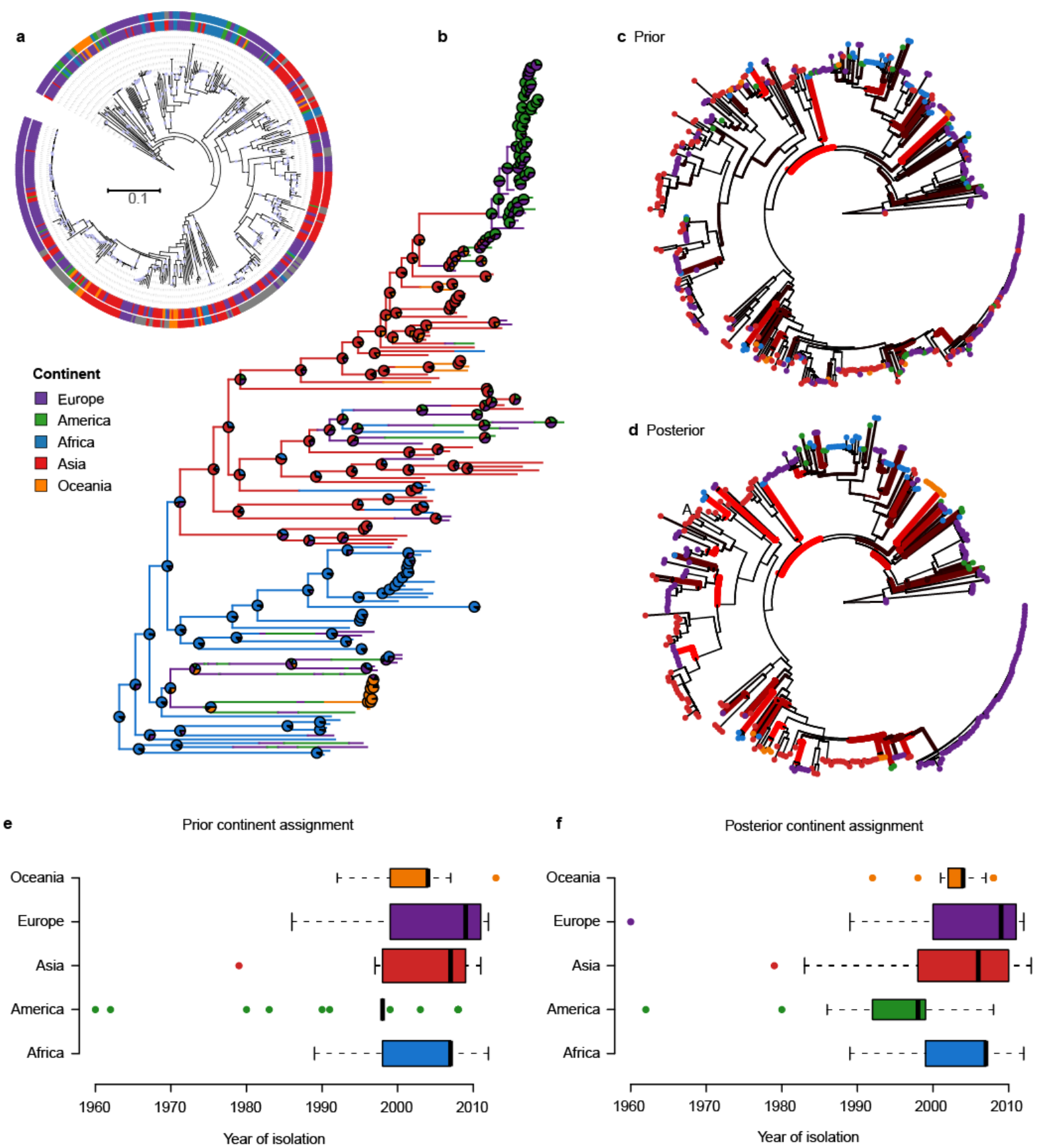

Extended Data Figure 3 | Phylogeographic analysis. (a) Maximum likelihood

phylogenetic tree showing the tips coloured with the prior information on the continent of isolation (inner circle) and the posterior group membership after DAPC analysis ${ }^{36}$ (outer circle). Admixed individuals are defined as those with $<80 \%$ posterior probability of assignment to any of the five continents and are shown in grey. Light blue circles represent bootstrap support values above 70\%. (b) Stochastic mapping of the posterior continent assignments over the dated phylogenetic tree complemented with US strains from Grad et al. 
$2014^{15}$ and using even number of strains from each continent $(\mathrm{N}=41)$ except for Oceania, for which there is not more available data in the public databases at the time of writing. Analysis was performed using the phytools $\mathrm{R}$ package $\mathrm{e}^{40}$. Pie charts in every node represent the proportion of ancestry to each of the five continents. (c-d) Per-branch posterior distribution of the trait "Continent" using both prior (c) and posterior (d) memberships along the phylogenetic tree calculated using treeBreaker ${ }^{41}$. (e-f) Distribution of isolation dates by continent shown for both prior (e) and posterior (f) continent assignments. The width of the boxplots is proportional to the number of strains from each continent. 


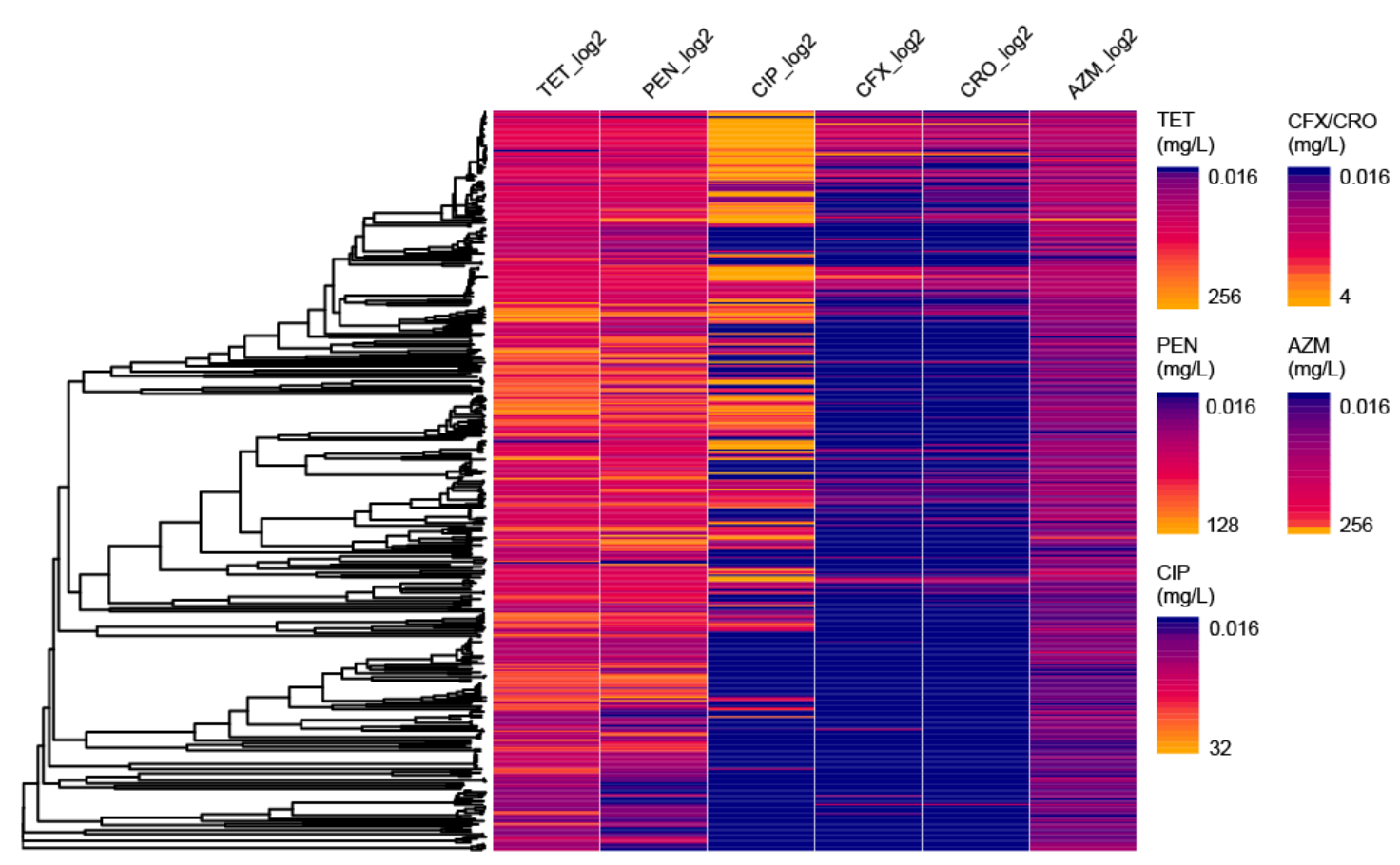

\section{Extended Data Figure 4 | Minimum Inhibitory Concentration (MIC, in mg/L)}

for six antimicrobials. The distribution of the MICs of six antimicrobials is shown

for all strains included in the analysis in $\log 2$ scale. Note that the distribution of resistance is equivalent to that found from genotypic resistance. The scale of the cephalosporins CFX and CRO has been collided into one and the range of MICs in the legend is shown without logging. The minimum MIC value has been set to 0.016 $\mathrm{mg} / \mathrm{L}$ for all antimicrobials. TET $=$ Tetracycline, $\mathrm{PEN}=$ Penicillin $\mathrm{G}, \mathrm{CIP}=$ Ciprofloxacin, $\mathrm{CFX}=$ Cefixime, $\mathrm{CRO}=$ Ceftriaxone, $\mathrm{AZM}=$ Azithromycin. 


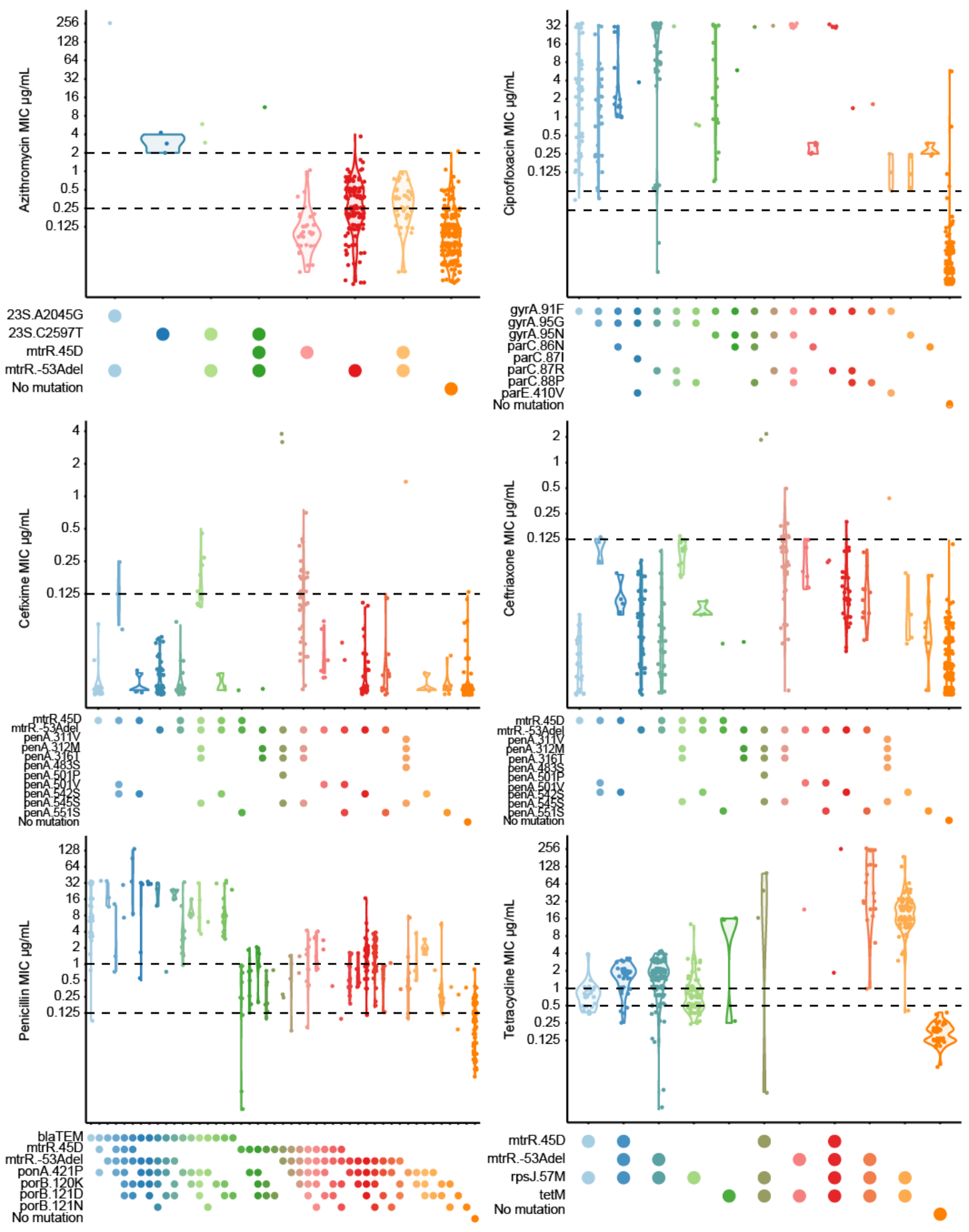

Extended Data Figure 5 | Phenotypic and genotypic resistance. Distribution of

MIC values (in logarithmic scale) for each combination of antimicrobial determinants associated in the literature ${ }^{42}$ to each of the six antimicrobials under study. Horizontal dashed lines mark EUCAST breakpoints (www.eucast.org) except for azithromycin, in which the CLSI $2 \mu \mathrm{g} / \mathrm{mL}$ upper bound is used. (www.clsi.org). 


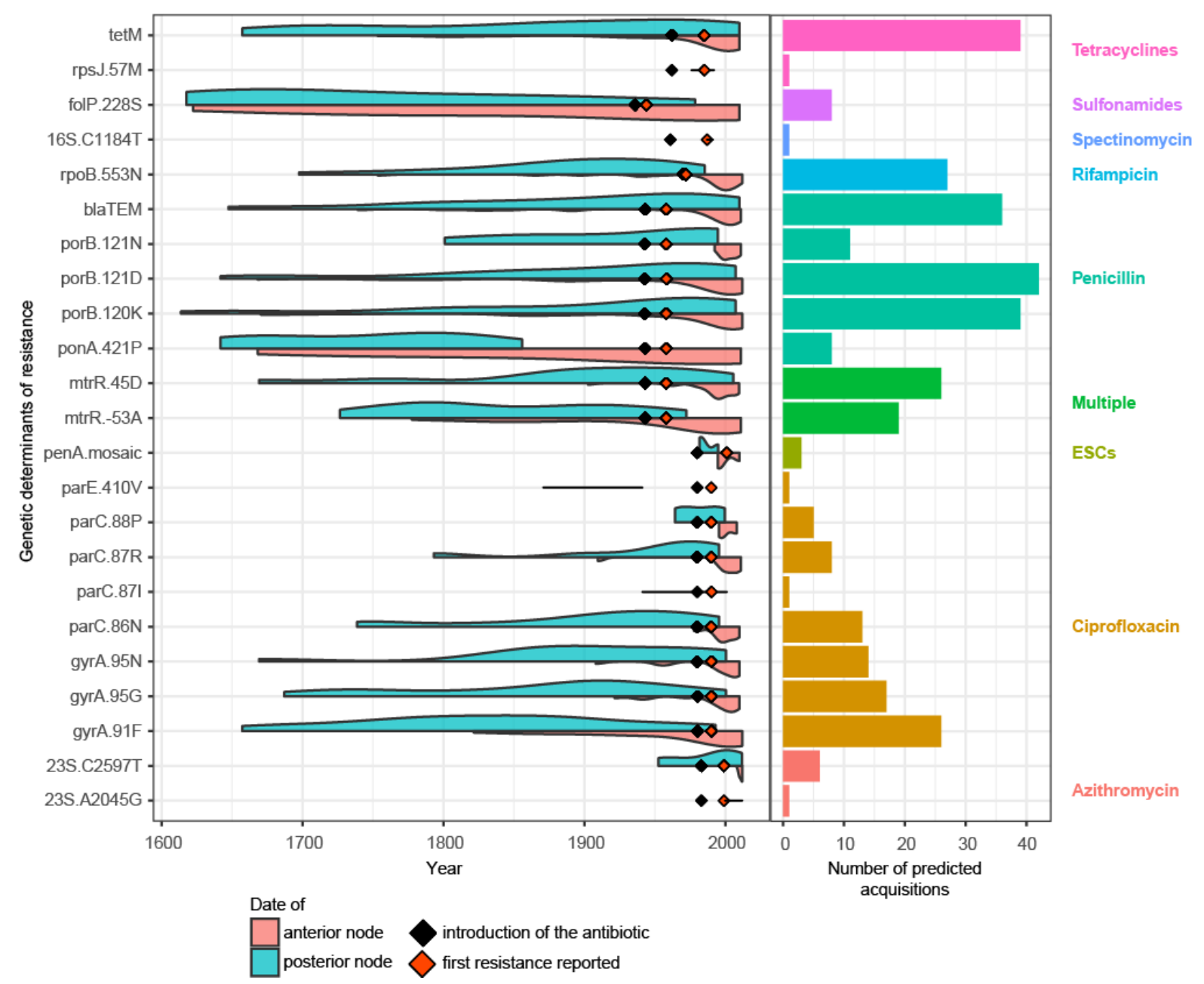

Extended Data Figure 6 | Most predicted acquisitions of antimicrobial resistance

happened after the introduction of antimicrobials. The distribution of the predicted acquisitions of the known determinants of antimicrobial resistance along time are represented as half violin plots (pink when using the date of the anterior and blue the posterior nodes of the branch where the acquisition is predicted). The prediction was performed with ancestral maximum likelihood reconstruction (ace function of the phytools $\mathrm{R}$ package $^{43}$ ) and a symmetric model of transition between states. Black diamonds mark the reported date of introduction of different antimicrobials to treat gonorrhoea and orange diamonds the first reported date of treatment failure. The barplot on the right shows the total number of predicted acquisitions for each mutation, and it is coloured to differentiate mutations affecting different antimicrobials. 


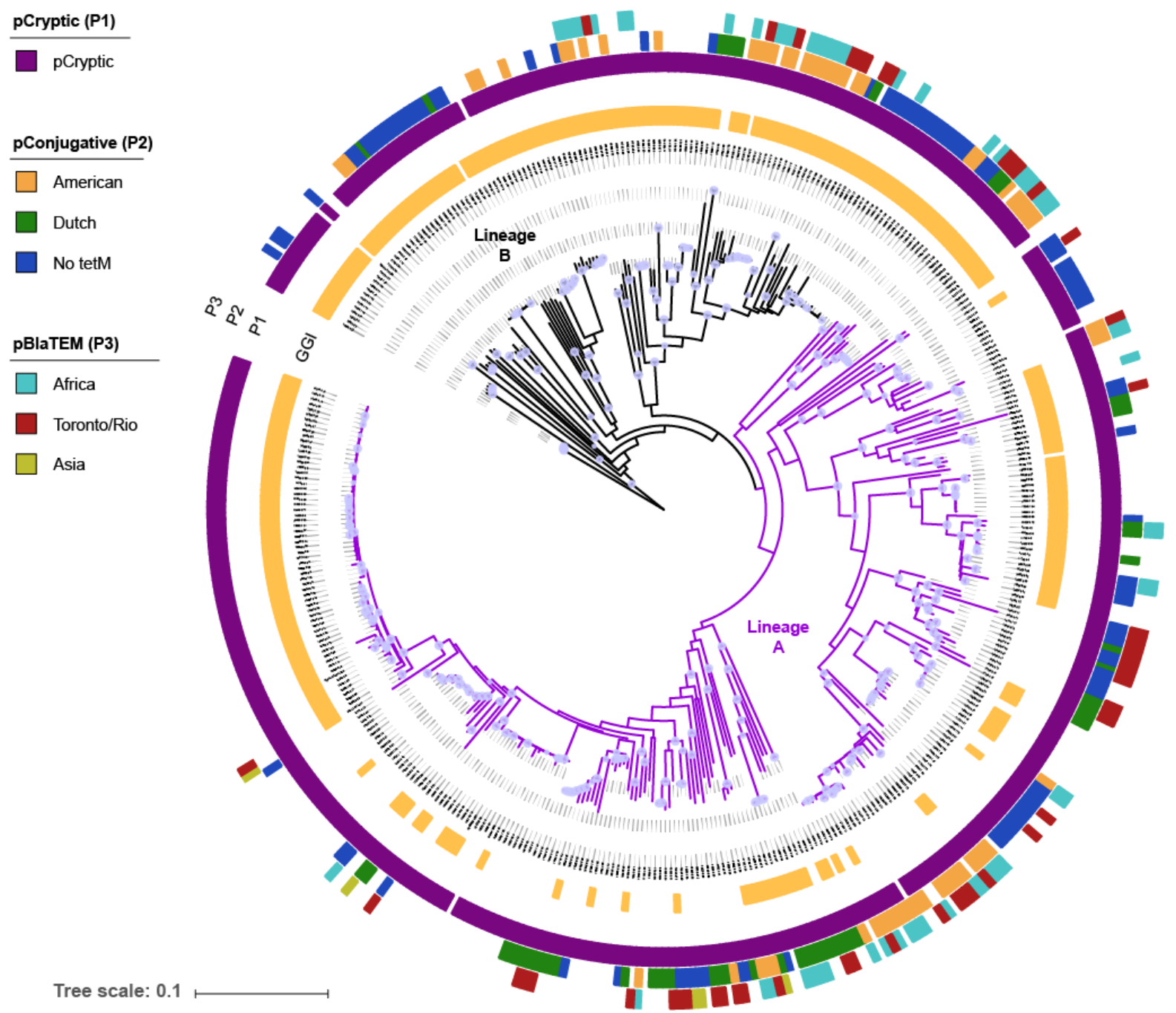

Extended Data Figure 7 | Distribution of the Gonococcal Genomic Island and plasmids.

Maximum likelihood phylogenetic tree showing, from the inner to the outer circle: the occurrence of the Gonococcal Genomic Island (GGI) and the three main plasmids (pCrypticP1, pConjugative-P2 and pBlaTEM-P3). Colours in plasmid tracks correspond to different types (see legend). The two lineages are marked in black and purple, respectively. Node shapes represent bootstrap support values above $70 \%$. 

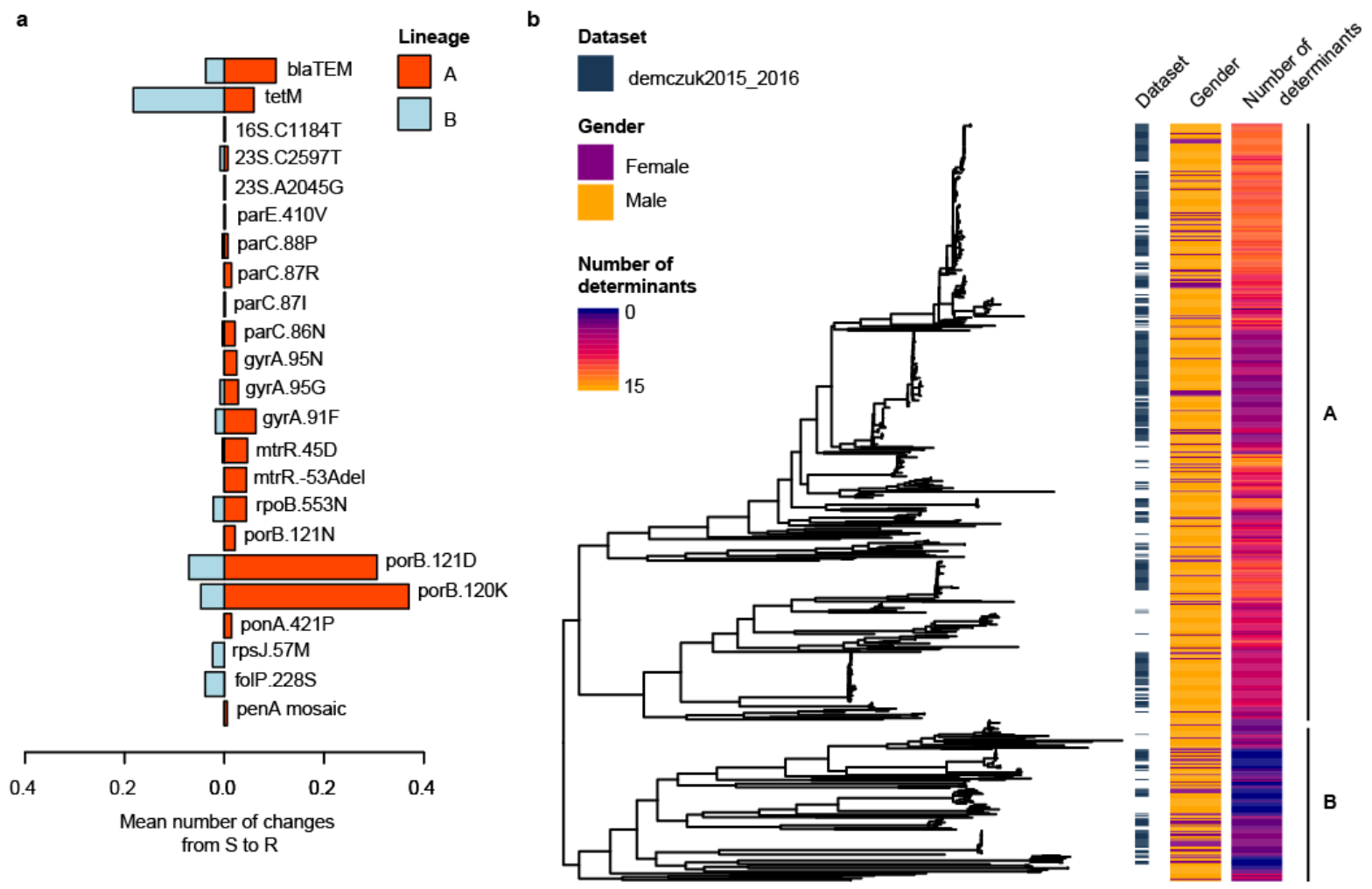

Extended Data Figure 8 | AMR genetic determinants in the two lineages. (a) Mean

number of changes from susceptible to antimicrobial resistant status inferred to have evolutionarily happened in lineages A and B for all the analysed resistance determinants, including the penA mosaic. Values have been corrected by the number of edges of the A ( N $=586)$ and $\mathrm{B}(\mathrm{N}=236)$ subtree lineages, respectively. (b) Distribution of the patient's gender and the number of antimicrobial resistance determinants that the infecting strains carry. The figure shows a non-recombinant maximum likelihood tree of 639 strains (263 from the global collection and 376 from two North American studies ${ }^{16,25}$ ) with the mentioned information as metadata. Lineages A and B are also labelled. 
bioRxiv preprint doi: https://doi.org/10.1101/334847; this version posted May 31, 2018. The copyright holder for this preprint (which was

not certified by peer review) is the author/funder, who has granted bioRxiv a license to display the preprint in perpetuity. It is made available under aCC-BY-NC-ND 4.0 International license.
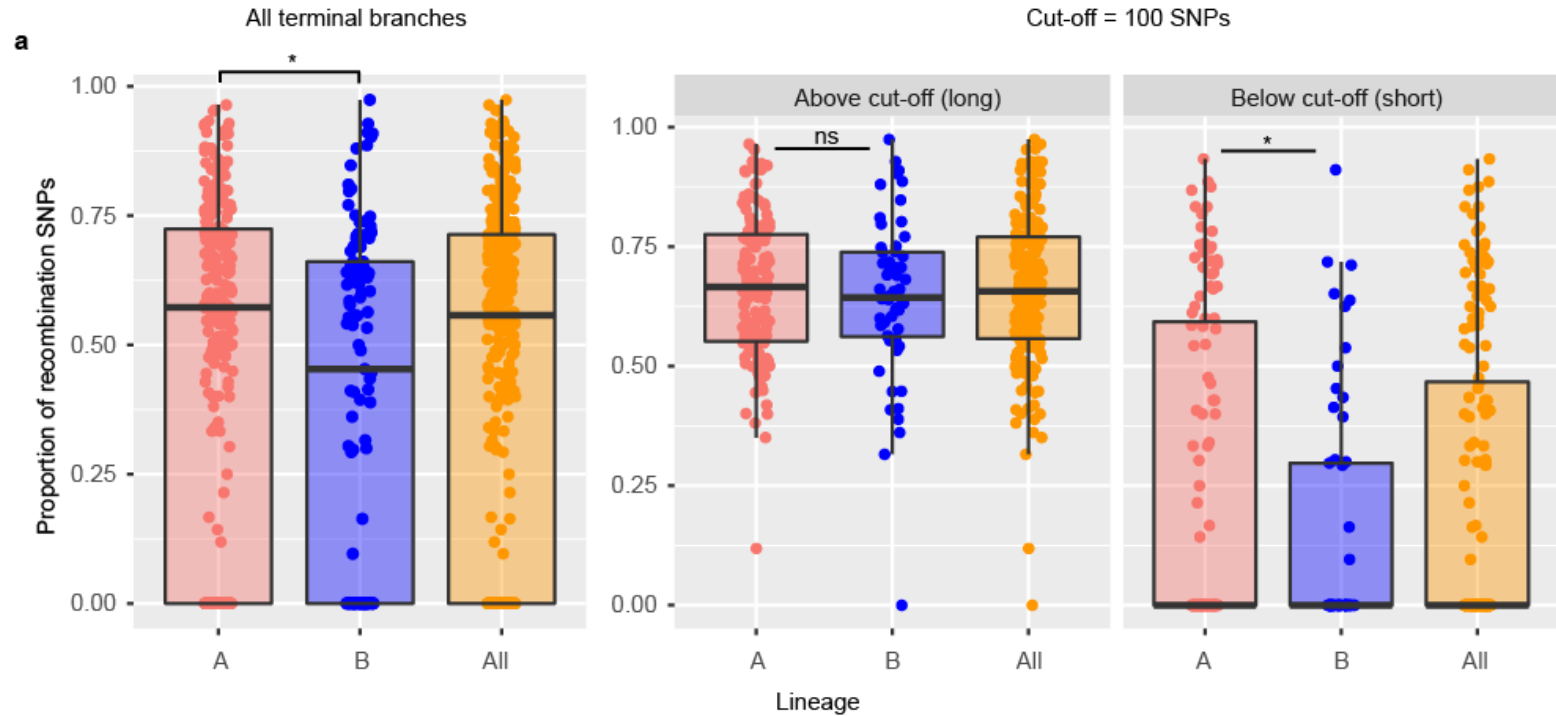

b
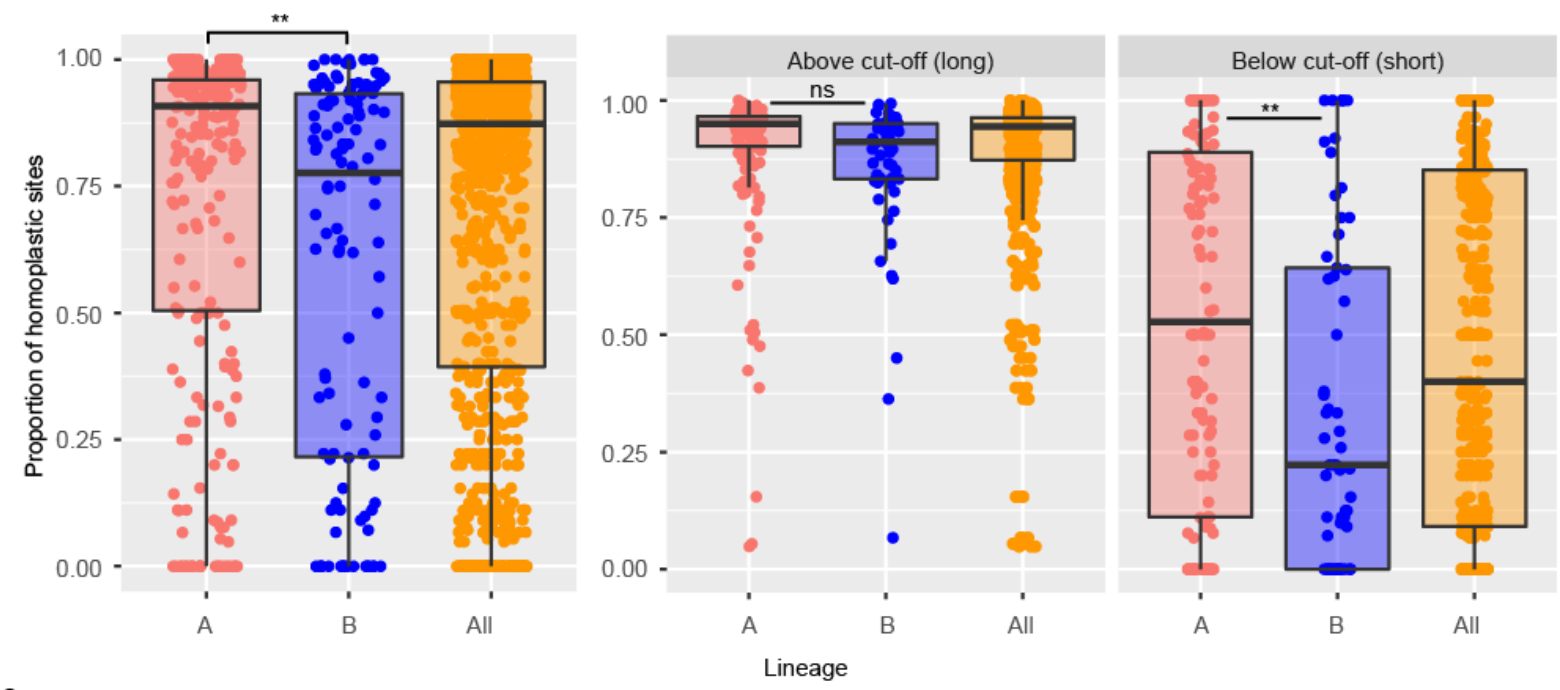

c

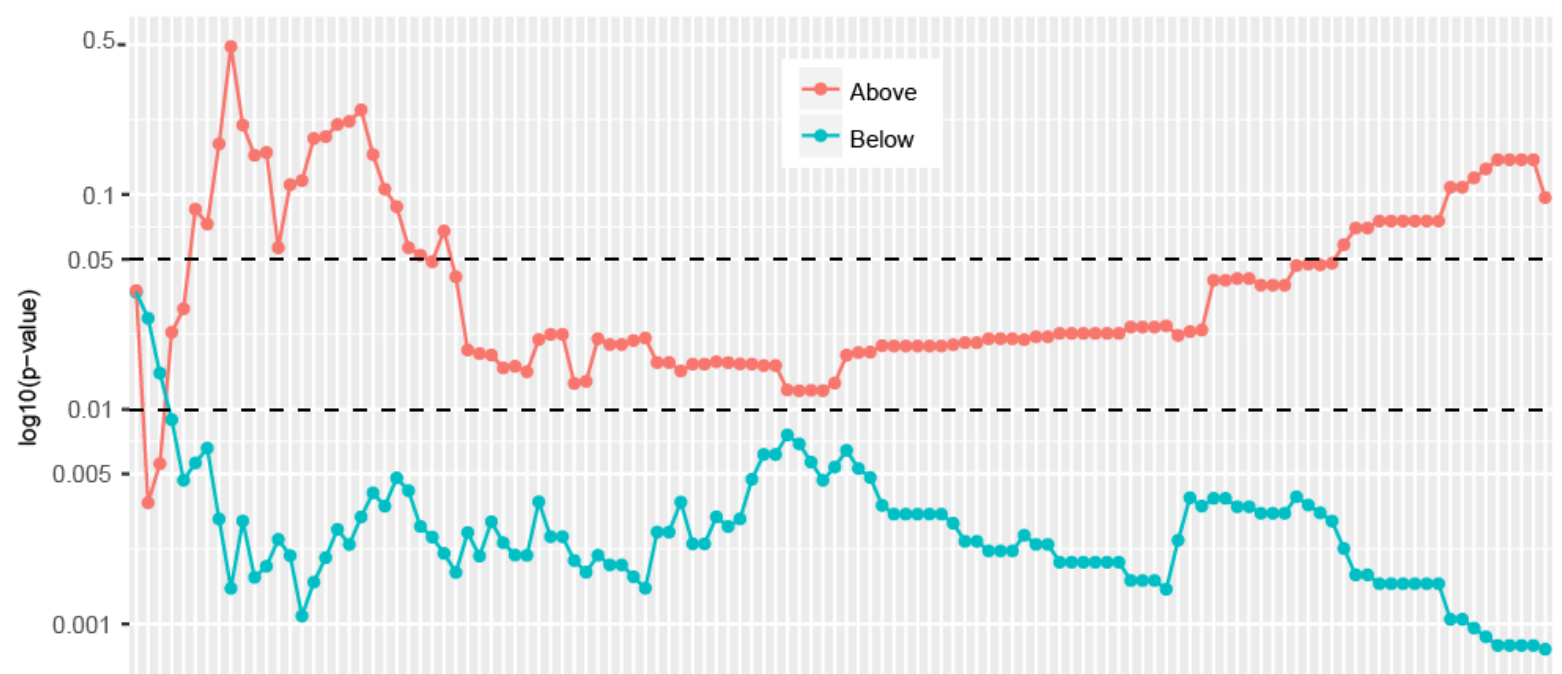

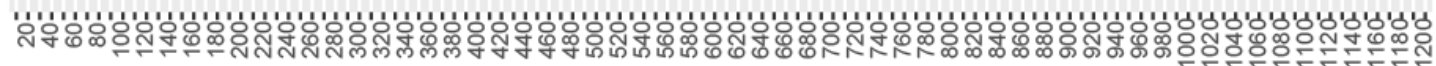




\section{Extended Data Figure 9 | Assessment of recombination SNPs and homoplasic sites in}

terminal branches. (a) Proportion of SNPs inside the recombination events predicted by

Gubbins for all terminal branches together and shorter (<=100 SNPs) and longer $(>100$

SNPs) terminal branches separately in lineages A and B and all strains. (b) Proportion of homoplasic sites in all terminal branches together and shorter $(<=100$ SNPs $)$ and longer

(>100 SNPs) terminal branches separately linesages A, B and all strains. (c) Distribution of the p-values calculated using a Student's t test on the number of homoplasies in the two lineages on short and long terminal branches of the tree at different SNP cut-offs.

Significance thresholds at 0.01 and 0.05 are marked with a dashed line. Short branches are probably more reliable for this type of calculation than long branches and this can be observed at around a cut-off of 100 SNPs, where branches under this number of SNPs have a p-value $<0.005$ while the rest are clearly not significant $p$-value $>0.1$. SNPs in repeat regions and those known to undergo antigenic variation, such as pilin-, opA54- or Maf-associated genes were excluded from the calculation. ${ }^{*}$ pvalue $<0.01,{ }^{*}$ - value $<0.05$, ns $=$ nonsignificant. 


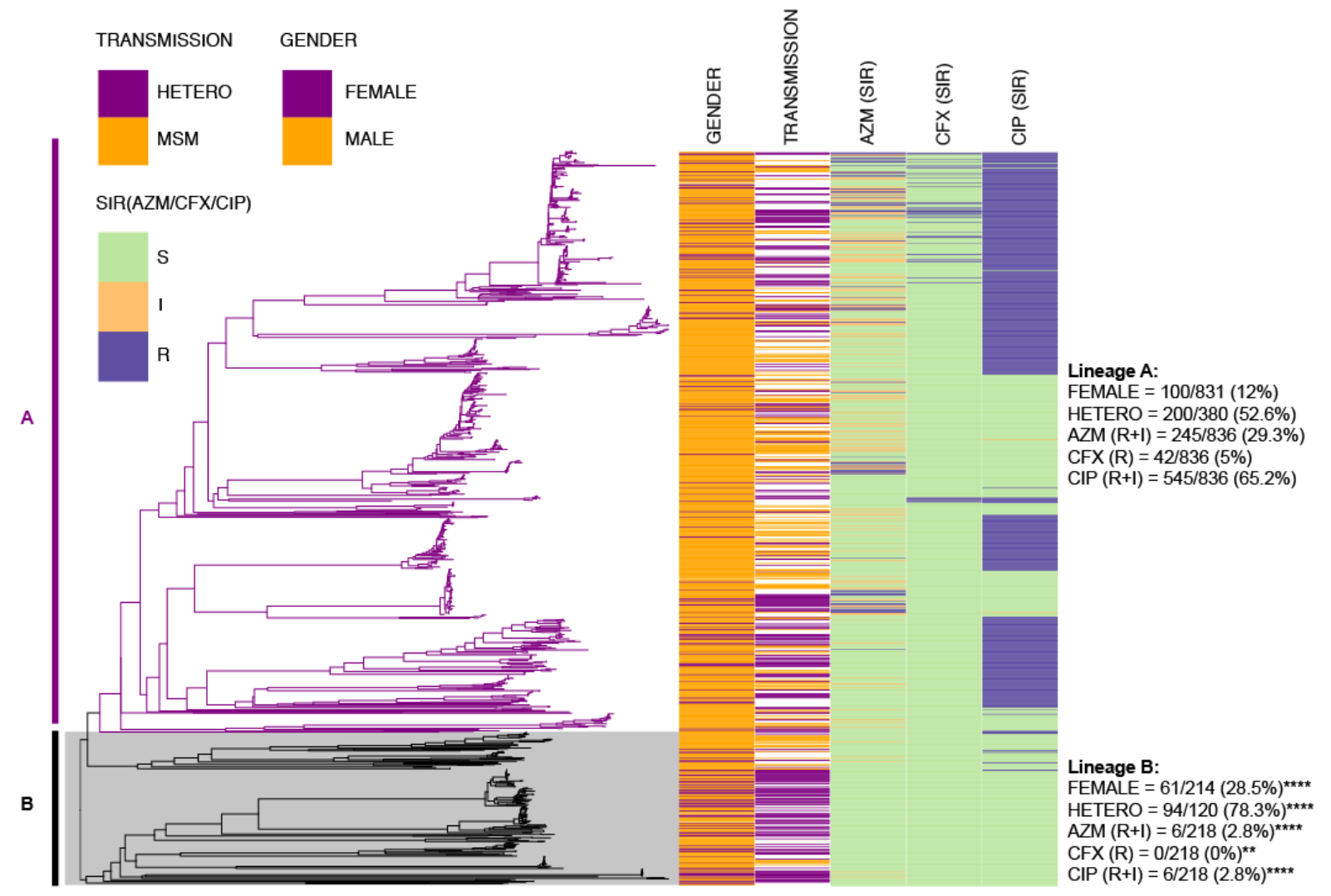

Extended Data Figure 10 | WGSA phylogenetic reconstruction of 1,054 strains from the

Euro-GASP 2013 survey $^{26}$. The two lineages (A in purple and B in black) were identified by combining this set with the global collection data as specified in the methods section. The metadata aligned to the tree shows the distribution of the gender of the patients from which the isolates were obtained, the type of transmission and the SIR categories of the phenotypic antimicrobial susceptibility test of the isolates following the breakpoints from EUCAST (www.eucast.org). Counts of each column are shown per lineage on the right side. Asterisks in lineage B indicate statistical significance compared to lineage A. AZM = Azithromycin, $\mathrm{CFX}=$ Cefixime, $\mathrm{CIP}=$ Ciprofloxacin $. \mathrm{R}=$ Resistant, $\mathrm{I}=$ Intermediate resistance, $\mathrm{S}=$ Susceptible. $* * * *$ p-value $<0.0001, * *$ p-value $<0.01$. 